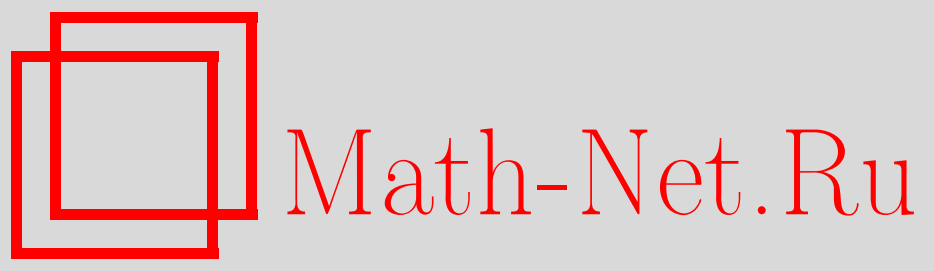

А. В. Мокшин, Самосогласованный подход к описанию релаксационных процессов в классических многочастичных системах, ТМФ, 2015, том 183, номер 1, 3-35

DOI: https://doi.org/10.4213/tmf8778

Использование Общероссийского математического портала Math-Net.Ru подразумевает, что вы прочитали и согласны с пользовательским соглашением http://www . mathnet.ru/rus/agreement

Параметры загрузки:

IP : 3.85 .183 .62

26 апреля 2023 г., 18:11:37

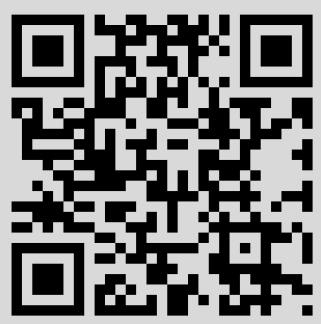




\section{САМОСОГЛАСОВАННЫЙ ПОДХОД К ОПИСАНИЮ РЕЛАКСАЦИОННЫХ ПРОЦЕССОВ В КЛАССИЧЕСКИХ МНОГОЧАСТИЧНЫХ СИСТЕМАХ}

Концепция временнь́х корреляционных функций представляет собой весьма удобный теоретический аппарат для описания релаксационных процессов в многочастичных системах. Это обусловлено тем, что, с одной стороны, корреляционные функции связываются непосредственно с экспериментально измеряемыми величинами (например, интенсивностями в спектроскопических измерениях, коэффициентами переноса через соотношения Кубо-Грина и т. д.), а с другой стороны, применимость данной концепции не ограничивается лишь равновесным случаем. Показано, что в рамках формализма функций памяти и метода рекуррентных соотношений возможно формулирование самосогласованного подхода к описанию релаксационных процессов в классических многочастичных системах, который исключает использование априорных аппроксимаций временнь́х корреляционных функций модельными зависимостями, обеспечивает выполнение правил сумм, а также других физических условий. Демонстрируется применимость подхода как при трактовке простейших релаксационных сценариев, так и в развитии микроскопических теорий транспортных явлений в жидкостях, распространения флуктуаций плотности в равновесных простых жидкостях и структурной релаксации в переохлажденных жидкостях. Подход обобщает приближения взаимодействующих мод в реализации Гётце-Левгезера и корреляционные приближения Юльметьева-Шурыгина.

Ключевые слова: релаксационные процессы, пространственно-временные корреляции, самосогласованное описание, приближение взаимодействующих мод, неупорядоченные системы, проекционные операторы, интегродифференциальные уравнения, рекуррентные соотношения.

DOI: $10.4213 / \operatorname{tmf} 8778$

Статья написана по заказу Редколлегии.

*Казанский (Приволжский) федеральный университет, Казань, Россия. E-mail: anatolii.mokshin@mail.ru

${ }^{\dagger}$ Институт теоретической физики им. Л. Д. Ландау РАН, Москва, Россия 


\section{СОДЕРЖАНИЕ}

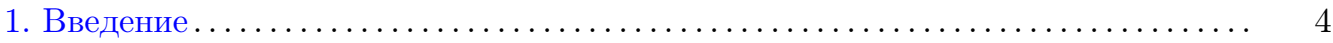

2. Проекционные операторы и динамические корреляции ................ 6

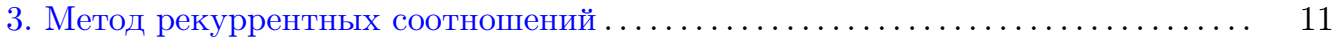

3.1. Основные рекуррентные соотношения ..................... 12

3.2. Непрерывная дробь и интегродифференциальные уравнения ....... 13

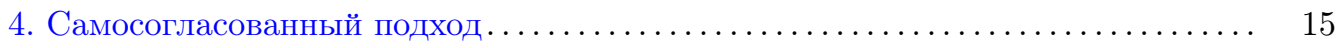

4.1. Конечный набор динамических переменных ................. 16

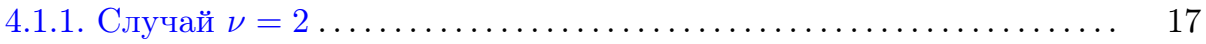

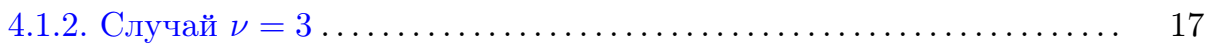

4.2. Бесконечный набор динамических переменных. Соотношения между временнб́ми масштабами ............................ 17

4.2 .1 Гауссова релаксация .................................. 18

4.2.2. Затухающий осциллирующий коррелятор................ 18

4.2.3. Коэффициенты переноса. Самодиффузия и плотность вибрационных состояний в равновесной простой жидкости ......... 19

4.2.4. Флуктуации плотности в равновесных простых жидкостях..... 22

4.3. Масштабируемость в релаксации....................... 27

4.3.1. Структурная релаксация в переохлажденных жидкостях. Приближения взаимодействующих мод $\ldots \ldots \ldots \ldots \ldots \ldots \ldots \ldots$

\section{1. ВВЕДЕНИЕ}

Системы, содержащие большое число взаимодействующих частиц, представляют собой основной предмет статистической механики, а описание таких систем как в равновесии, так и в неравновесии - ее основную задачу [1]. Среди разнообразия методов, применяемых к описанию динамики многочастичных систем, достаточно результативными, простыми и удобными оказываются методы, основанные на применении двухвременнь́х опережающих и запаздывающих функций Грина, введенных Боголюбовым и Тябликовым [2]-[9], а также методы, использующие математический аппарат временнь́х корреляционных функций (BКФ) [10]-[13], распределений, моментов и кумулянтов [14]-[17]. В настоящей работе мы не будем обсуждать все разнообразие методов, существующих для описания динамики многочастичных систем, - это, конечно же, трудноосуществимо, и такая задача не ставится, мы ограничимся рассмотрением подхода, основу которого формирует концепция ВКФ.

Весьма важным является то, что ВКФ позволяют естественным образом реализовать статистико-вероятностное рассмотрение кинетики системы. При этом многие величины, измеряемые экспериментально, выражаются через ВКФ от соответствующих переменных. В качестве примера можно привести коэффициенты переноса (вязкость, диффузию, теплопроводность), а также отклики, измеряемые в опытах по диэлектрической, оптической, нейтронной, рентгеновской спектроскопии, ядерному магнитному резонансу. Так, Цванциг [11] указывает на то, что значимость ВКФ в решении задач неравновесной статистической механики сопоставима со значимостью статистических сумм и пространственных парных корреляционных 
функций, поскольку многие свойства могут быть выражены непосредственно через ВКФ (см. с. 7 в монографии [11]). В своем высказывании Цванциг отчасти солидарен с Берне и Харпом [18], которые отмечают более развернуто: “... временны́е корреляционные функции сделали для теории неравновесных процессов то же самое, что статистические суммы для равновесной теории. Задача стала хорошо определенной, но, тем не менее, не стала более легкой для решения. Хотя мы знаем, какая корреляционная функция соответствует тому или иному конкретному явлению, тем не менее расчет ВКФ остается крайне сложным"1) (с. 67 в [18]). Несмотря на то что это утверждение было высказано более сорока лет назад, актуальность его все еще сохраняется, причиной чему служит отсутствие общего строгого алгоритма для расчета ВКФ [2]. В результате этого возникает необходимость в применении различных теоретических приближений к рассматриваемым ВКФ, в основу которых закладываются предположения о некотором поведении ВКФ, воспроизводимом модельными функциями: например, экспоненциальной, гауссовой [19], гиберболическим секансом [20], комбинациями этих и других модельных функций. Такие приближения лежат в основе вязкоупругой модели Лавси [19], обобщенной гидродинамической модели [21], приближения обобщенных коллективных мод [22]. В этих приближениях выполняется аппроксимация либо исходной ВКФ, либо некоторой ВКФ, появляющейся на определенном уровне бесконечной цепочки кинетических уравнений, что по сути эквивалентно выполнению обрыва этой цепочки.

Хотя упомянутые выше приближения позволяют предложить некоторую интерпретацию экспериментальных результатов, отсутствие явного малого параметра (как, например, в случае любых плотных жидкостей для негидродинамической области) затрудняет получение весомых физических аргументов, обосновывающих применимость подобных приближений. Ввиду этого вполне понятный интерес может представлять другой, отчасти более строгий подход, в котором поведение ВКФ и/или ее спектральных особенностей определяется самосогласованным образом [23], [24], исключающим аппроксимирование ВКФ модельными функциональными зависимостями. Основу такого подхода образуют теоретические модели, развиваемые в рамках метода рекуррентных соотношений [14], [25]-[28], приближений взаимодействующих (связанных) мод [29], [30], корреляционных приближений ЮльметьеваШурыгина [31]-[34]. Характерно, что данный подход формирует единую основу для развития микроскопических теорий различных релаксационных процессов в многочастичных системах. В настоящей работе ограничимся рассмотрением простых жидкостей, под которыми, следуя Френкелю [39], будем подразумевать однокомпонентные системы, где частицы “идентичны" и взаимодействуют через сферический потенциал ${ }^{2)}$.

В разделе 2 приводится общий вывод кинетических интегродифференциальных уравнений в рамках техники проекционных операторов. В разделе 3 обсуждается метод рекуррентных соотношений, который, с одной стороны, может рассматриваться как альтернатива техники проекционных операторов, а с другой стороны, может

1)Здесь Берне и Харп подразумевают прежде всего аналитический расчет ВКФ.

2) Под частицами будем понимать такие структурные элементы системы, как атомы, молекулы, частицы коллоидного раствора и т. д. [35], динамика которых удовлетворяет классическому описанию [21], [36]-[38]. 
выступать в качестве основы к общему самосогласованному подходу описания релаксационных процессов в многочастичных системах. Данный подход представлен в разделе 4, где также демонстрируется его применение к трактовке простейших релаксационных сценариев (таких как гауссова релаксация, затухающий осциллирующий коррелятор), к развитию микроскопических теорий для расчета коэффициентов переноса, описанию динамики частиц в равновесных и переохлажденных простых жидкостях.

\section{2. ПРОЕКЦИОННЫЕ ОПЕРАТОРЫ И ДИНАМИЧЕСКИЕ КОРРЕЛЯЦИИ}

Рассмотрим систему, состоящую из $N$ идентичных классических частиц массы $m$, находящихся в некотором объеме $V$ с количественной плотностью $n=N / V$. Koординаты и скорости частиц $\left(\mathbf{r}^{(l)}\right.$ и $\mathbf{v}^{(l)}$, где $\left.l=1,2, \ldots, 3 N\right)$ образуют $6 N$-мерное фазовое пространство

$$
\mathcal{A}\left(\mathbf{r}^{(1)}, \ldots, \mathbf{r}^{(l)}, \mathbf{v}^{(1)}, \ldots, \mathbf{v}^{(l)}\right),
$$

на котором определяются динамические переменные. В качестве динамической переменной может быть выбрана, например, величина, характеризующая траекторию частицы

$$
\rho_{s}(\mathbf{r}, t)=\delta\left(\mathbf{r}-\mathbf{r}_{s}(t)\right),
$$

скорость частицы $\mathbf{v}_{s}(t)$ или энергию частицы [40]

$$
e_{s}(t)=\frac{m\left|\mathbf{v}_{s}\right|^{2}}{2}+\frac{1}{2} \sum_{s \neq j}^{N} U\left(r_{s j}\right)
$$

( $m$ - масса частицы, $U(r)$ - потенциал межчастичного взаимодействия), локальную плотность

$$
\delta \rho(k)=\frac{1}{\sqrt{N}} \sum_{j=1}^{N} e^{i \mathbf{k} \cdot \mathbf{r}_{j}}
$$

(k - волновой вектор), локальные параметры порядка [41]

$$
q_{l}(s)=\left(\frac{4 \pi}{2 l+1} \sum_{m=-l}^{l}\left|\frac{1}{n_{b}(s)} \sum_{j=1}^{n_{b}(s)} Y_{l m}\left(\Omega_{s j}\right)\right|^{2}\right)^{1 / 2}, \quad l=6,8,10, \ldots
$$

$\left(n_{b}(s)\right.$ - число частиц в пределах первой координационной сферы для частицы $\left.s[42]\right)$ или локальные сдвиговые напряжения

$$
\sigma_{x y}^{(\mathcal{N})}=\sum_{i=1}^{\mathcal{N}}\left(m v_{i x} v_{i y}+\frac{1}{2} \sum_{i \neq j}^{\mathcal{N}} F_{i j x} r_{i j y}\right)
$$

$(\mathcal{N}$ - число частиц, характеризующих локальную область, где $\mathcal{N} \leqslant N)$. 
В свою очередь, гамильтониан $\widehat{H}$ определяет операцию усреднения $\langle A\rangle$ через плотность распределения фазового пространства $\rho \propto e^{-(\widehat{H}-\mu N) /\left(k_{\mathrm{B}} T\right)}$, где $\mu-$ химический потенциал. Тогда в гильбертовом пространстве можно определить скалярное произведение с помощью набора динамических переменных:

$$
(A, B)=\frac{\left\langle A^{*} B\right\rangle}{k_{\mathrm{B}} T}, \quad\langle A\rangle=\langle B\rangle=0 .
$$

Кроме того, гамильтониан определяет временну́ю эволюцию, отображая $A \rightarrow A(t)$ через каноническое уравнение движения

$$
\frac{d A(t)}{d t}=i\{\widehat{H}, A(t)\}=i \widehat{\mathcal{L}} A(t),\left.\quad A \equiv A(t)\right|_{t=0}
$$

где $\{\widehat{H}, \cdot\}$ - скобки Пуассона, а оператор Лиувилля

$$
\widehat{\mathcal{L}}=-i \sum_{j} \frac{\mathbf{p}_{j}}{m} \cdot \frac{\partial}{\partial \mathbf{r}_{j}}-i \sum_{j} \mathbf{F}_{j} \frac{\partial}{\partial \mathbf{p}_{j}}
$$

является эрмитовым. Формальное решение уравнения (8) имеет следующий вид:

$$
A(t)=e^{i \widehat{\mathcal{L}} t} A
$$

где $e^{i \widehat{\mathcal{L}} t}-$ оператор эволюции (или пропагатор), величина $\mathbf{F}_{j}$ - полная сила, действующая на $j$-ю частицу. Для сохранения общности подхода не будем связывать переменную $A$ с какой-либо конкретной физической величиной на данном этапе изложения.

Определим ВКФ двух переменных следующим образом:

$$
\phi_{A B}(t, \tau)=\left\langle A^{*}(\tau) B(t+\tau)\right\rangle .
$$

Эрмитовость оператора Лиувилля $\widehat{\mathcal{L}}$ устанавливает следующее тождество:

$$
(\widehat{\mathcal{L}} A, B) \equiv(B, \widehat{\mathcal{L}} A)
$$

из которого непосредственно следуют некоторые свойства ВКФ [21]. Прежде всего, используя соотношение (12), можно показать, что в случае стационарных процессов справедливо равенство

$$
\begin{aligned}
\left\langle A^{*}(\tau) B(t+\tau)\right\rangle & =k_{\mathrm{B}} T\left(e^{-i \widehat{\mathcal{L}} \tau} A, e^{i \widehat{\mathcal{L}}(t+\tau)} B\right)= \\
& =k_{\mathrm{B}} T\left(A, e^{i \widehat{\mathcal{L}} t} B\right)=\left\langle A^{*}(0) B(t)\right\rangle .
\end{aligned}
$$

Далее, из тождества (12) следует

$$
\phi_{A \dot{B}}=k_{\mathrm{B}} T(A, i \widehat{\mathcal{L}} B)=-k_{\mathrm{B}} T(i \widehat{\mathcal{L}} A, B)=-\phi_{\dot{A} B}
$$

Ограничимся рассмотрением стационарного случая, для которого определим нормированную временну́ю автокорреляционную функцию

$$
\phi_{A A}(t)=\frac{\left\langle A^{*}(0) A(t)\right\rangle}{\left\langle|A(0)|^{2}\right\rangle} \equiv \frac{\left\langle A^{*}(0) e^{i \widehat{\mathcal{L}} t} A(0)\right\rangle}{\left\langle|A(0)|^{2}\right\rangle},
$$


обладающую следующими свойствами:

$$
\begin{gathered}
\left.\phi_{A A}(t)\right|_{t=0}=1, \\
1 \geqslant\left|\phi_{A A}(t)\right| \geqslant 0, \\
\left.\frac{d \phi_{A A}(t)}{d t}\right|_{t=0}=0 .
\end{gathered}
$$

Используя теорему Винера-Хинчина [43], можно показать, что спектр

$$
\tilde{\phi}_{A A}(\omega)=\int_{-\infty}^{+\infty} d t e^{-i \omega t} \phi_{A A}(t)
$$

является действительным и положительным, $\tilde{\phi}(\omega) \geqslant 0$.

Разлагая в ряд оператор временно́й эволюции в правой части уравнения (10)

$$
A(t)=\left(1+i \widehat{\mathcal{L}} t-\frac{1}{2} \widehat{\mathcal{L}}^{2} t^{2}+\cdots\right) A(0),
$$

получим коротковременное разложение для ВКФ

$$
\phi_{A A}(t)=1-\frac{1}{2 !} \omega^{(2)} t^{2}+\frac{1}{4 !} \omega^{(4)} t^{4}-\frac{1}{6 !} \omega^{(6)} t^{6}+\mathcal{O}\left(t^{8}\right),
$$

где $\omega^{(p)}$ - частотные моменты порядка $p$ :

$$
\omega^{(2 p)}=\left.(-i)^{p} \frac{d^{p} \tilde{\phi}_{A A}(t)}{d t^{p}}\right|_{t=0}, \quad p=1,2, \ldots
$$

Определим проекционные операторы

$$
\Pi_{0}=\frac{\left.A_{0}(0)\right\rangle\left\langle A_{0}^{*}(0)\right.}{\left\langle\left|A_{0}(0)\right|^{2}\right\rangle}, \quad P_{0}=1-\Pi_{0},
$$

обладающие следующими свойствами:

$$
\begin{gathered}
\Pi_{0}+P_{0}=1, \\
\Pi_{0}^{2}=\Pi_{0}, \quad P_{0}^{2}=\left(1-\Pi_{0}\right)^{2}=P_{0}, \\
\Pi_{0} A_{0}(0)=A_{0}(0), \\
\Pi_{0} A_{0}(t)=A_{0}(0) F(t), \\
\Pi_{0} P_{0}=\Pi_{0}\left(1-\Pi_{0}\right)=P_{0} \Pi_{0}=0 .
\end{gathered}
$$

Подействуем слева на уравнение Лиувилля (8) операторами $\Pi_{0}$ и $P_{0}$. Для удобства введем обозначения

$$
A_{0}^{\prime}(t)=\Pi_{0} A_{0}(t), \quad A_{0}^{\prime \prime}(t)=P_{0} A_{0}(t) .
$$

Тогда уравнение Лиувилля (10) примет следующий вид:

$$
\begin{aligned}
\frac{d}{d t} \Pi_{0} A_{0}(t) & =\frac{d}{d t} A_{0}^{\prime}(t)=i \widehat{\mathcal{L}}_{11}^{0} A_{0}^{\prime}(t)+i \widehat{\mathcal{L}}_{12}^{0} A_{0}^{\prime \prime}(t), \\
\frac{d}{d t} P_{0} A_{0}(t)=\frac{d}{d t} A_{0}^{\prime \prime}(t) & =i \widehat{\mathcal{L}}_{21}^{0} A_{0}^{\prime}(t)+i \widehat{\mathcal{L}}_{22}^{0} A_{0}^{\prime \prime}(t),
\end{aligned}
$$


где введены обозначения

$$
\begin{array}{cc}
\widehat{\mathcal{L}}_{11}^{0}=\Pi_{0} \widehat{\mathcal{L}} \Pi_{0}, \quad \widehat{\mathcal{L}}_{12}^{0}=\Pi_{0} \widehat{\mathcal{L}} P_{0}, \quad \widehat{\mathcal{L}}_{21}^{0}=P_{0} \widehat{\mathcal{L}} \Pi_{0}, \quad \widehat{\mathcal{L}}_{22}^{0}=P_{0} \widehat{\mathcal{L}} P_{0} . \\
\widehat{\mathcal{L}}_{11}^{0}+\widehat{\mathcal{L}}_{12}^{0}+\widehat{\mathcal{L}}_{21}^{0}+\widehat{\mathcal{L}}_{22}^{0}=\widehat{\mathcal{L}} .
\end{array}
$$

Применяя оператор преобразования Лапласа $\widehat{L T}$

$$
\widehat{L T}[f(\tau)]=\int_{0}^{\infty} e^{-s \tau} f(\tau) d \tau=\tilde{f}(s)
$$

ко второму уравнению системы (30), получаем

$$
\widetilde{A}_{0}^{\prime \prime}(s)=\frac{A_{0}^{\prime \prime}(0)}{s-i \widehat{\mathcal{L}}_{22}^{0}}+\frac{i \widehat{\mathcal{L}}_{21}^{0} \widetilde{A}_{0}^{\prime}(s)}{s-i \widehat{\mathcal{L}}_{22}^{0}} .
$$

Выполняя обратное преобразование Лапласа в уравнении (34), находим

$$
A_{0}^{\prime \prime}(t)=e^{i \widehat{\mathcal{L}}_{22}^{0} t} A_{0}^{\prime \prime}(0)+i \int_{0}^{t} d \tau e^{i \widehat{\mathcal{L}}_{22}^{0} \tau} \widehat{\mathcal{L}}_{21}^{0} A^{\prime}(t-\tau) .
$$

Согласно свойству (26) имеем

$$
A_{0}^{\prime \prime}(0)=P_{0} A_{0}(0)=\left(1-\Pi_{0}\right) A_{0}(0)=0 .
$$

Подставляя уравнение (35) в первое уравнение системы (30), с учетом соотношения (36) получаем следующее интегродифференциальное уравнение:

$$
\begin{aligned}
\frac{d}{d t} A_{0}^{\prime}(t) & =i \widehat{\mathcal{L}}_{11}^{0} A_{0}^{\prime}(t)-\widehat{\mathcal{L}}_{12}^{0} \int_{0}^{t} d \tau e^{i \widehat{\mathcal{L}}_{22}^{0} \tau} \widehat{\mathcal{L}}_{21}^{0} A_{0}^{\prime}(t-\tau)= \\
& =i \widehat{\mathcal{L}}_{11}^{0} A_{0}^{\prime}(t)-\int_{0}^{t} d \tau \widehat{\mathcal{L}}_{12}^{0} e^{i \widehat{\mathcal{L}}_{22}^{0} \tau} \widehat{\mathcal{L}}_{21}^{0} A_{0}^{\prime}(t-\tau)
\end{aligned}
$$

Представляя оператор $\Pi_{0}$ в виде

$$
\left.\Pi_{0}=R_{0} S_{0}, \quad R_{0}=A_{0}(0)\right\rangle, \quad S_{0}=\frac{\left\langle A_{0}^{*}(0)\right.}{\left\langle\left|A_{0}(0)\right|^{2}\right\rangle},
$$

определяем свойства оператора $S_{0}$ :

$$
S_{0} A_{0}(0)=1, \quad S_{0} A_{0}(t)=\phi_{A A}(t), \quad S_{0} R_{0}=1, \quad S_{0} \Pi_{0}=S_{0} .
$$

Последовательно подействуем оператором $S_{0}$ слева на каждую из трех частей выражения (37). Тогда левая часть выражения (37) принимает вид

$$
S_{0} \frac{d}{d t} A_{0}^{\prime}(t)=\frac{d \phi_{A A}(t)}{d t}
$$

первый член в правой части равенства (37)

$$
i S_{0} \widehat{\mathcal{L}}_{11}^{0} A_{0}^{\prime}(t)=i S_{0} \widehat{\mathcal{L}} R_{0} \phi_{A A}(t) .
$$


Подынтегральное выражение в (37) есть

$$
S_{0} \widehat{\mathcal{L}}_{12}^{0} e^{i \widehat{\mathcal{L}}_{22}^{0} \tau} \widehat{\mathcal{L}}_{21}^{0} A_{0}^{\prime}(t-\tau)=\frac{\left\langle\left(A_{0}(0) \widehat{\mathcal{L}}\right)^{*} e^{i \widehat{\mathcal{L}}_{22}^{0} \tau}\left(\widehat{\mathcal{L}} A_{0}(0)\right)\right\rangle}{\left\langle\left|A_{0}(0)\right|^{2}\right\rangle}-\left[\frac{\left\langle A_{0}^{*}(0) \widehat{\mathcal{L}} A_{0}(0)\right\rangle}{\left\langle\left|A_{0}(0)\right|^{2}\right\rangle}\right] .
$$

Находим уравнение движения

$$
\frac{d A_{0}(t)}{d t}=-\int_{0}^{\infty} A_{0}(t-\tau) \frac{\left\langle(i \widehat{\mathcal{L}} A(0))^{*} A_{1}(\tau)\right\rangle}{\left\langle\left|A_{0}(0)\right|^{2}\right\rangle} d \tau+A_{1}(t)
$$

которое для ВКФ принимает вид кинетического интегродифференциального уравнения

$$
\frac{d \phi_{0}(t)}{d t}=-\Delta_{1} \int_{0}^{t} \phi_{1}(\tau) \phi_{0}(t-\tau) d \tau, \quad \phi_{0}(t) \equiv \phi_{A A}(t)
$$

Величина

$$
A_{1}(t)=i \widehat{\mathcal{L}} A_{0}(t)
$$

есть вторая динамическая переменная, ортогональная к $A_{0}:\left(A_{0}, A_{1}\right)=0$. Соотношение

$$
\Delta_{1}=\frac{\left\langle\left|A_{1}(0)\right|^{2}\right\rangle}{\left\langle\left|A_{0}(0)\right|^{2}\right\rangle}
$$

определяет так называемый первый частотный параметр, имеющий размерность квадрата частоты. Как видно из (42) и (43), явный вид частотного параметра $\Delta_{1}$ зависит от того, какая величина выбрана в качестве исходной переменной $A_{0}$. И, наконец, величина

$$
\phi_{1}(\tau)=\frac{\left\langle A_{1}^{*}(0) e^{i \widehat{\mathcal{L}}_{22}^{0} \tau} A_{1}(0)\right\rangle}{\left\langle\left|A_{1}(0)\right|^{2}\right\rangle}
$$

есть так называемая функция памяти первого порядка, которая представляет собой ВКФ переменной $A_{1}$ и полностью удовлетворяет свойствам (16)-(18).

Последовательность процедур (29)-(41) представляет собой стандартный вывод кинетического интегродифференциального уравнения для ВКФ $\phi_{0}(t)$ из уравнения движения для динамической переменной $A_{0}$ с помощью техники проекционных операторов. Здесь существенным является то, что уравнение (41) получено точно. Впервые такой вывод был представлен в работах Цванцига [44] и Мори [45], а подобная реализация техники проекционных операторов в дальнейшем стала обозначаться как формализм функций памяти (или формализм Цванцига-Мори) [21]. Отметим, что величина $A_{1}$ также является динамической переменной, определенной на фазовом пространстве $(1)$, и ее эволюция задается соответствующим уравнением Лиувилля вида (8). Тогда, определяя проекционные операторы для $A_{1}$ аналогично (23) и повторяя последовательность действий (29)-(41), получим кинетическое интегродифференциальное уравнение для ВКФ $M_{1}(t)$, в которое, в свою очередь, будет входить функция памяти второго порядка $\phi_{2}(t)$, являющаяся ВКФ новой переменной $A_{2}$, ортогональной $A_{0}$ и $A_{1}$.

Техника проекционных операторов позволяет осуществить разделение "собственного движения" рассматриваемой динамической переменной $A_{0}$ и эффектов взаимодействия $A_{0}$ с ортогональной ей “потоковой” переменной, динамика которой связана с другими степенями свободы. Так, например, в случае, когда $A_{0}$ представляет 
собой скорость движения частицы, величина $A_{1}$ соотносится со "стохастической силой”, оказывающей влияние на движение частицы посредством ее взаимодействия с окружением. Величина $A_{1}$ непосредственно входит в уравнение движения для исходной динамической переменной $A_{0}$. Другой вклад, который также определяет эволюцию $A_{0}$, учитывает коррелированность в динамике $A_{1}$ и ее взаимодействие с $\mathrm{BK} \Phi \phi_{0}(t)$. В соответствии с такой формулировкой поведение $A_{0}(t)$ может становиться более упорядоченным в результате взаимодействий между $A_{0}$ и $A_{1}$, которые происходили в предыдущие моменты времени. Механизм этого вклада, управляющий поведением системы и вносящий вклад в диссипацию, связывается с так называемой статистической памятью [11], [46], [47].

В результате описание процесса осуществляется в ортогональном базисе

$$
\mathbf{A}=\left\{A_{0}, A_{1}, A_{2}, \ldots, A_{\nu}, \ldots\right\},
$$

где переменные удовлетворяют свойству

$$
\left\langle A_{\nu} A_{\mu}\right\rangle=\left\{\begin{array}{ll}
0, & \nu \neq \mu, \\
\left\langle\left|A_{\nu}\right|^{2}\right\rangle, & \nu=\mu,
\end{array} \quad \nu, \mu=0,1, \ldots,\right.
$$

а процедура конструирования базиса идентична процедуре ортогонализации Грама-Шмидта [43]. Переменные набора А связаны цепочкой интегродифференциальных уравнений

$$
\begin{aligned}
\frac{d A_{\nu}(t)}{d t} & =-\Delta_{\nu+1} \int_{0}^{\infty} A_{\nu}(t-\tau) \frac{\left.\left\langle A_{\nu+1}(0)\right)^{*} A_{\nu+1}(\tau)\right\rangle}{\left\langle\left|A_{\nu+1}(0)\right|^{2}\right\rangle} d \tau+A_{\nu+1}(t), \\
\Delta_{\nu+1} & =\frac{\left\langle\left|A_{\nu+1}(0)\right|^{2}\right\rangle}{\left\langle\left|A_{\nu}(0)\right|^{2}\right\rangle}
\end{aligned}
$$

которой соответствует цепочка кинетических интегродифференциальных уравнений

$$
\frac{d \phi_{\nu}(t)}{d t}=-\Delta_{\nu+1} \int_{0}^{\infty} \phi_{\nu}(t-\tau) \phi_{\nu+1}(\tau) d \tau .
$$

Здесь исходный рассматриваемый процесс, соотносимый с переменной $A_{0}$, перестает выступать в качестве обособленного и характеризуется взаимосвязью с другими процессами, протекающими в системе. Особенно наглядно это реализуется в методе рекуррентных соотношений [25], [26], предложенном Ли [27].

\section{3. МЕТОД РЕКУРРЕНТНЫХ СООТНОШЕНИЙ}

Важной особенностью метода рекуррентных соотношений является то, что динамика системы здесь непосредственно связана с особенностями реализуемого гильбертова пространства, содержащего конкретную физическую информацию [27], [47]. Так, например, поведение рассматриваемой динамической переменной непосредственно определяется размерностью пространства, его формой и структурными характеристиками. Подобные или близкие идеи высказывались в работах Боголюбова [48], [49], Зубарева [12], Кубо [13]. 
3.1. Основные рекуррентные соотношения. Пусть пространство $\mathcal{S}$ является реализацией абстрактного гильбертова пространства [25], [26], определяемого скалярным произведением, известным также как произведение Кубо [13]:

$$
(X, Y)=k_{\mathrm{B}} T \int_{0}^{\beta}\left\langle e^{\lambda \widehat{H}} Y^{\dagger} e^{-\lambda \widehat{H}} X\right\rangle d \lambda,
$$

где $X, Y \subset \mathcal{S}$, а величина $(X, Y)$ называется релаксационной функцией переменных $X$ и $Y$. Здесь $\langle X Y\rangle$ - усреднение по ансамблю, определяемое формулой

$$
\langle X Y\rangle=\frac{\operatorname{Tr}\left[X Y e^{-\widehat{H} / k_{\mathrm{B}} T}\right]}{\operatorname{Tr}\left[e^{-\widehat{H} / k_{\mathrm{B}} T}\right]} .
$$

Динамическая переменная $A_{0}(t)$ является вектором этого пространства и характеризуется нормой $\left\|A_{0}(t)\right\|=\left(A_{0}(t), A_{0}(t)\right)$. Если система эрмитова, то норма не изменяется при движении по поверхности пространства $\mathcal{S}$, т. е. $\left\|A_{0}(t)\right\|=\left\|A_{0}\right\|$. Таким образом, $A_{0}(t)$ может менять лишь направление, но неизменной остается длина $\left\|A_{0}(t)\right\|$. С ростом $t$ вектор $A_{0}(t)$ будет вычерчивать на поверхности пространства $\mathcal{S}$ траекторию, определяемую свойствами этого пространства [25].

Объем пространства $\mathcal{S}$ определяется полным набором ортогональных базис-векторов $\left\{f_{0}, f_{1}, \ldots, f_{d-1}\right\}$,

$$
\left(f_{\nu}(k), f_{\mu}(k)\right)=\left(f_{\nu}(k), f_{\nu}(k)\right) \delta_{\nu \mu}, \quad \nu, \mu=0,1, \ldots, d-1,
$$

которые связаны так называемым первым рекуррентным соотношением

$$
\begin{aligned}
f_{\nu+1} & =i \mathcal{L} f_{\nu}+\Delta_{\nu} f_{\nu-1}, \\
\Delta_{\nu} & =\frac{\left(f_{\nu}, f_{\nu}\right)}{\left(f_{\nu-1}, f_{\nu-1}\right)}, \quad \nu=0,1, \ldots, d-1, \quad f_{-1}=0, \quad \Delta_{0} \equiv 1 .
\end{aligned}
$$

Величина $d$ определяет размерность пространства. Базис-векторы зависят от общих свойств скалярного произведения для данного пространства и, следовательно, являются физическими характеристиками системы.

В произвольный момент времени $t$ вектор $A_{0}(t)$ имеет проекции на каждый базисный вектор $f_{\nu}$. Обозначим размер проекции вектора $A_{0}(t)$ на $\nu$-й базисный вектор в момент времени $t$ через $\phi^{(\nu)}(t)$ :

$$
\phi^{(\nu)}(t)=\frac{\left(A_{0}(t), f_{\nu}\right)}{\left(f_{\nu}, f_{\nu}\right)} .
$$

Следуя работе [27], для $A_{0}(t)$ можно записать ортогональное разложение

$$
A_{0}(t)=\sum_{\nu=0}^{d-1} \phi^{(\nu)}(t) f_{\nu}
$$

и определить начальное условие для динамической переменной

$$
f_{0}=\left.A_{0}(t)\right|_{t=0}=A_{0}
$$


Тогда из уравнения (55) получаем следующие граничные условия для $\left.\phi^{(\nu)}(t)\right|_{t=0}$ :

$$
\left.\phi^{(\nu)}(t)\right|_{t=0}= \begin{cases}1, & \nu=0 \\ 0, & \nu=1,2, \ldots\end{cases}
$$

Сопоставляя выражения (7), (15) и (54), находим, что $\phi^{(0)}(t)$ отождествляется с релаксационной функцией

$$
\phi^{(0)}(t) \equiv \phi_{0}(t)
$$

где

$$
\phi_{0}(t)=\frac{\left(A_{0}(t), f_{0}\right)}{\left(f_{0}, f_{0}\right)}=\frac{\left(A_{0}(t), A_{0}(0)\right)}{\left(A_{0}(0), A_{0}(0)\right)} .
$$

Тогда из (53) и (59) можно получить так называемое второе рекуррентное coотношение [25], [26]

$$
\Delta_{\nu+1} \phi^{(\nu+1)}(t)=-\frac{d \phi^{(\nu)}(t)}{d t}+\phi^{(\nu-1)}(t), \quad \nu=0,1, \ldots, d-1, \quad \phi^{(-1)} \equiv 0 .
$$

Таким образом, если первое рекуррентное соотношение (53) устанавливает связь между динамическими переменными, то второе соотношение (60) указывает на связь между динамиками различных релаксационных процессов, протекающих в системе.

3.2. Непрерывная дробь и интегродифференциальные уравнения. Применяя оператор преобразования Лапласа $\widehat{L T}$ ко второму рекуррентному соотношению (60), можно получить два уравнения

$$
\begin{aligned}
1 & =s \tilde{\phi}^{(0)}(s)+\Delta_{1} \tilde{\phi}^{(1)}(s), \\
\tilde{\phi}^{(\nu-1)}(s) & =s \tilde{\phi}^{(\nu)}(s)+\Delta_{\nu+1} \tilde{\phi}^{(\nu+1)}(s), \quad \nu=0,1, \ldots, d-1,
\end{aligned}
$$

совместное решение которых дает непрерывную дробь

$$
\tilde{\phi}^{(0)}(s) \equiv \tilde{\phi}_{0}(s)=\frac{1}{s+\frac{\Delta_{1}}{s+\frac{\Delta_{2}}{s+\frac{\Delta_{3}}{s+\cdots}}}} .
$$

Следуя работе [25], можно определить следующую величину:

$$
\tilde{\psi}^{(\nu)}(s)=\frac{\tilde{\phi}^{(\nu)}(s)}{\tilde{\phi}^{(0)}(s)}, \quad \nu=1,2, \ldots, d-1 .
$$

Из соотношений (61) с учетом выражения (63) получаем

$$
\tilde{\phi}^{(0)}(s)=\frac{1}{s+\Delta_{1} \tilde{\psi}^{(1)}(s)} .
$$


Тогда из (64) и (62) находим

$$
\tilde{\psi}^{(1)}(s) \equiv \tilde{\phi}_{1}(s)=\frac{1}{s+\frac{\Delta_{2}}{s+\frac{\Delta_{3}}{s+\frac{\Delta_{4}}{s+\cdots}}}} .
$$

Применяя обратное преобразование Лапласа к выражению (64), получаем

$$
\frac{d \phi^{(0)}(t)}{d t}+\Delta_{1} \int_{0}^{t} d \tau \psi^{(1)}(t-\tau) \phi^{(0)}(\tau)=0
$$

Уравнение (66) соответствует первому интегродифференциальному уравнению цепочки (49) (см. также уравнение $(41)$ ), где $\psi^{(1)}(t)$ соотносится с функцией памяти первого порядка $\phi_{1}(t)$,

$$
\phi_{1}(t) \equiv \psi^{(1)}(t)
$$

Функция $\psi^{(1)}$ представляет собой проекцию переменной $A_{1}(t)$ на базис-вектор $f_{1}$,

$$
\psi^{(1)}(t)=\frac{\left(A_{1}(t), f_{1}\right)}{\left(f_{1}, f_{1}\right)}=\frac{\left(A_{1}(t), A_{1}(0)\right)}{\left(A_{1}(0), A_{1}(0)\right)},
$$

где

$$
f_{1}=\left.A_{1}(t)\right|_{t=0}=A_{1}
$$

По аналогии с разложением (55) можно записать разложение для переменной $A_{1}(t)[25],[26]:$

$$
A_{1}(t)=\sum_{\nu=1}^{d-1} \psi^{(\nu)}(t) f_{\nu}
$$

где функция $\psi^{(\nu)}(t)$ определяет размер проекции вектора $A_{1}$ на базис $f_{\nu}$ в момент времени $t$,

$$
\psi^{(\nu)}(t)=\frac{\left(A_{1}(t), f_{\nu}\right)}{\left(f_{\nu}, f_{\nu}\right)}, \quad \nu=1,2, \ldots,
$$

а также удовлетворяет следующим граничным условиям:

$$
\left.\psi^{(\nu)}(t)\right|_{t=0}= \begin{cases}1, & \nu=1 \\ 0, & \nu=2,3, \ldots\end{cases}
$$

Таким образом, если динамика переменной $A_{0}$ происходит в пространстве $\mathcal{S}$, образованном базис-векторами $f_{0}, f_{1}, \ldots, f_{d-1}$, то динамика переменной $A_{1}$ - в подпространстве $\mathcal{S}_{1}$, натянутом на базис-векторы $f_{1}, f_{2}, \ldots, f_{d-1}$, и $\mathcal{S}_{1} \perp f_{0}$.

Представление величины $\tilde{\phi}_{0}(s)$ в виде непрерывной дроби (62), получаемое в методе рекуррентных соотношений, идентично представлению в частотном лаплас-отображении цепочки кинетических интегродифференциальных уравнений (49), получаемых в формализме Цванцига-Мори (см. раздел 2). 


\section{4. САМОСОГЛАСОВАННЫЙ ПОДХОД}

Формализм Цванцига-Мори указывает на возможность осуществления описания релаксационных процессов, протекающих в многочастичных системах, с помощью системы интегродифференциальных уравнений. Тем не менее какого-либо общего алгоритма решения этих уравнений данный формализм не предоставляет. В результате этого вплоть до настоящего времени появляются различные теоретические приближения, в основу которых изначально закладываются предположения о некотором поведении ВКФ, воспроизводимом модельными функциями: экспоненциальной [45], гауссовой [19], гиперболическим секансом [20], комбинациями этих и других модельных функций. Подобные приближения составляют основу вязкоупругой модели Лавси [19], так называемой обобщенной гидродинамической модели [50], подхода обобщенных коллективных мод [22]. Здесь вполне естественно возникает вопрос: возможно ли развитие общего самосогласованного подхода в нахождении ВКФ $\phi_{\nu}(t)$ без угадывания и аппроксимирования релаксационного поведения какими-либо модельными зависимостями? Необходимость в развитии такого подхода является понятной, поскольку априорные приближения релаксационного поведения ВКФ $\phi_{\nu}(t)$ зачастую нарушают физические принципы, например, такие как:

1) спектральные характеристики ВKФ должны удовлетворять правилам сумм [51];

2) ВКФ должна принимать конечные значения, что следует из (16)-(18);

3) корректное и строгое описание динамики возможно в ортогональном базиce [31], [43].

С другой стороны, метод рекуррентных соотношений (см. раздел 3) указывает на то, что динамика исследуемой системы полностью определяется частотными параметрами $\Delta_{\nu}$ (в частности, структурными характеристиками, определяющими значения частотных параметров). Таким образом, задача о нахождении исходной $\mathrm{BK} \Phi \phi_{0}(t)$ может быть сведена к аналитическому расчету частотных параметров $\Delta_{\nu}$, оценке их значений. Поскольку частотные параметры непосредственно связаны с динамическими переменными (выражение (48)), то их количество и значения определяются физическими условиями, в которых реализуется релаксационный процесс, соотносимый с динамической переменной $A_{0}$. Так, например, как видно из (62), высокочастотные свойства должны быть более выражены в функциях памяти высоких порядков [47]. Можно выделить три класса физических ситуаций, когда реализация самосогласованности в описании является возможной.

1. Набор динамических переменных $\mathbf{A}=\left\{A_{0}, A_{1}, \ldots, A_{\nu}\right\}$ изначально является конечным. Такая ситуация соответствует конечному набору временны́х масштабов $\tau_{\nu-1}=\Delta_{\nu}^{-1 / 2}$

$$
\Delta_{1}^{-1 / 2}, \quad \ldots, \quad \Delta_{\nu-1}^{-1 / 2} \neq 0, \quad \Delta_{\nu}^{-1 / 2}=0,
$$

и является наиболее простой. В этом случае решения, получаемые для функции $\phi_{0}(t)$, будут описываться незатухающими гармоническими зависимостями. Некоторые соответствующие этому классу решения рассмотрены в работах Ли (см., например, [27]).

2. Набор динамических переменных $\mathbf{A}=\left\{A_{0}, A_{1}, \ldots, A_{\nu}, \ldots\right\}$ характеризуется определенными соотношениями между временны́ми масштабами $\tau_{\nu}=\Delta_{\nu}^{-1 / 2}$, где 
$\nu=0,1, \ldots$ и $\Delta_{\nu}^{-1 / 2} \neq 0$ при любом $\nu$. Соотношения между временнь́ми масштабами обеспечиваются термодинамическими условиями [52] (например, рассматриваемой областью фазовой диаграммы, размером характеристического пространственного масштаба и т. д.), структурными особенностями системы [53], [54] или другими причинами [55], [56]. В данном случае решения могут характеризовать самое разнообразное релаксационное (как правило, затухающее) поведение. Впервые на возможность такой реализации обратил внимание Боголюбов [48], [49], сформулировавший постулат об иерархии времен релаксации в конденсированных средах. При этом частный случай выравнивания временны́х масштабов различных релаксационных процессов приводит к прямой реализации идеи Боголюбова о сокращенном описании. Корреляционные приближения, предложенные Юльметьевым [33], [34], формируют некоторые теоретические модели в рамках данного класса. Следует отметить, что нахождение релаксационной функции также возможно в рамках данного класса, когда известно соотношение между временнь́ми масштабами лишь некоторой группы динамических переменных набора А. В этом случае полное поведение функции $\phi_{0}(t)$ может характеризоваться сложными (нетривиальными) временны́ми зависимостями и выражаться в виде функционала от частотных параметров:

$$
\phi_{\nu}(t)=\mathcal{F}\left[\Delta_{\nu}, \Delta_{\nu+1}, \Delta_{\nu+2}, \ldots\right]
$$

Конкретными примерами релаксационных процессов данного класса являются процессы, связанные с микроскопической одночастичной и коллективной динамикой в равновесных жидкостях [47], [57], [58].

3. В релаксации различных процессов, соотносимых с динамическими переменными набора $\mathbf{A}=\left\{A_{0}, A_{1}, \ldots, A_{\nu}, \ldots\right\}$, наблюдается подобие

$$
\phi_{\nu}(t) \propto \phi_{\mu}(t)^{p}, \quad \nu, \mu=0,1, \ldots, \quad p>0 .
$$

Здесь определение функции $\phi_{0}(t)$ возможно также в случае, когда полная релаксация системы происходит на временно́м масштабе, значительно превышающем рассматриваемый, а подобие в релаксации выполняется лишь для отдельных этапов. Такое свойство позволяет получать решения для квазиравновесных и неравновесных ситуаций, когда релаксация системы происходит на временно́м масштабе, превышающем рассматриваемый (экспериментально наблюдаемый). Типичным примером реализации такой ситуации служит структурная релаксация в переохлажденных жидкостях и стеклах вблизи стеклования [29], [30], [59]-[61].

В настоящей работе рассмотрены примеры, соответствующие всем трем приведенным выше классам.

4.1. Конечный набор динамических переменных. Рассмотрим случай с конечным набором динамических переменных, т. е. когда $d$ конечно. Такая ситуация реализуется при условии $A_{\nu}=0$ и $\Delta_{\nu}=0$ и соответствует неэргодическим процессам, описываемым незатухающими, осциллирующими ВКФ. 
4.1.1. Случай $\nu=2$. При условии $\nu=2^{3)}$ имеем $A_{2}=0$ и $\Delta_{2}=0$. Здесь непрерывная дробь (62) преобразуется в систему двух уравнений

$$
\begin{aligned}
1-s \tilde{\phi}_{0}(s) & =\Delta_{1} \tilde{\phi}_{0}(s) \tilde{\phi}_{1}(s), \\
s \tilde{\phi}_{1}(s) & =1,
\end{aligned}
$$

которая имеет простые решения

$$
\begin{aligned}
& \phi_{0}(t)=\cos \left(\Delta_{1}^{1 / 2} t\right), \\
& \phi_{1}(t)=1 .
\end{aligned}
$$

Корреляционная функция вида (74) воспроизводит поведение незатухающего гармонического осциллятора. Такая ситуация реализуется, например, в случае флуктуаций плотности однородного электронного газа при конечных волновых числах $k$ и температуре $T=0$ [28]. Другим примером, где такой случай уместен, является динамика цепочки классических гармонических осцилляторов. Здесь временна́я автокорреляционная функция скорости осцилляторов, $\phi_{0}(t)=\langle\boldsymbol{v}(0) \cdot \boldsymbol{v}(t)\rangle /\langle\boldsymbol{v}(0) \cdot \boldsymbol{v}(0)\rangle$, описывается выражением (74) [62].

4.1.2. Случай $\nu=3$. В данном случае справедливы условия $A_{3}=0$ и $\Delta_{3}=0$. Тогда дробь (62) преобразуется в систему уравнений следующего вида:

$$
\begin{aligned}
1-s \tilde{\phi}_{0}(s) & =\Delta_{1} \tilde{\phi}_{0}(s) \tilde{\phi}_{1}(s), \\
1-s \tilde{\phi}_{1}(s) & =\Delta_{2} \tilde{\phi}_{1}(s) \tilde{\phi}_{2}(s), \\
s \tilde{\phi}_{2}(s) & =1,
\end{aligned}
$$

которая может быть решена с помощью обратного преобразования Лапласа. В результате получаем решения

$$
\begin{aligned}
\phi_{0}(t) & =\frac{1}{\Delta_{1}+\Delta_{2}}\left[\Delta_{2}+\Delta_{1} \cos \left(\sqrt{\Delta_{1}+\Delta_{2}} t\right)\right] \\
\phi_{1}(t) & =\cos \left(\Delta_{2}^{1 / 2} t\right), \\
\phi_{2}(t) & =1 .
\end{aligned}
$$

Выражение (77) снова соответствует гармоническому поведению ВКФ $\phi_{0}(t)$, период осцилляций которого определяется частотными параметрами $\Delta_{1}$ и $\Delta_{2}$ [14].

Из двух рассмотренных случаев очевидно, что точное аналитическое решение для корреляционных функций $\phi_{\nu}(t)$ может быть получено при любом конечном значении $\nu$.

4.2. Бесконечный набор динамических переменных. Соотношения между временны́ми масштабами. В случае, когда динамические переменные $\mathbf{A}=$ $\left\{A_{0}, A_{1}, \ldots, A_{\nu}, \ldots\right\}$ формируют бесконечный набор, возможно разнообразное поведение $\phi_{\nu}(t)$. Универсальным в этом случае является затухающий характер автокорреляций.

\footnotetext{
3) Случай $\nu=1$ является тривиальным.
} 
4.2.1. Гауссова релаксация. Учитывая, что частотные параметры $\Delta_{\nu}$ характеризуют квадратичный релаксационный масштаб $\tau_{\nu-1}=\Delta_{\nu}^{-1 / 2}$, рассмотрим случай, когда частотные параметры $\Delta_{\nu}$ связаны арифметической прогрессией:

$$
\Delta_{1}, \quad \Delta_{2}=2 \Delta_{1}, \quad \Delta_{3}=3 \Delta_{1}, \quad \ldots, \quad \Delta_{\nu}=\nu \Delta_{1}
$$

Тогда непрерывная дробь (62) принимает вид

$$
\tilde{\phi}_{0}(s)=\frac{1}{s+\frac{\Delta_{1}}{s+\frac{2 \Delta_{1}}{s+\frac{3 \Delta_{1}}{s+\cdots}}}},
$$

что есть во временно́м отображении представление гауссовой функции [63]

$$
\phi_{0}(t)=e^{-\Delta_{1} t^{2} / 2}
$$

В качестве наиболее известного примера здесь можно привести релаксацию флуктуаций плотности в идеальном газе, а также релаксацию, связанную с одночастичной динамикой в равновесных жидкостях [21], [64].

С другой стороны, точное соответствие между частотными параметрами, устанавливаемое соотношениями (80), указывает на возможность количественной оценки отклонения релаксационного процесса от гауссовой зависимости с помощью простого сопоставления параметров $\Delta_{\nu}$ :

$$
\alpha_{\nu}=\frac{\nu}{\nu+1} \frac{\Delta_{\nu+1}}{\Delta_{\nu}}-1
$$

В случае гауссовой релаксации имеем $\alpha_{\nu}=0$, в то время как отклонения параметров $\alpha_{\nu}$ от нулевых значений будут характеризовать негауссово поведение корреляционной функции $\phi_{0}(t)[65]$.

4.2.2. Затухающий осциллирующий коррелятор. Рассмотрим частный случай, когда частотные параметры являются конечными и принимают одинаковые значения, что связывается с выравниванием характеристических временнь́х масштабов:

$$
\Delta_{1}^{-1 / 2}=\Delta_{2}^{-1 / 2}=\Delta_{3}^{-1 / 2}=\cdots=\Delta_{\nu}^{-1 / 2} .
$$

В этом случае непрерывная дробь (62) принимает вид

$$
\tilde{\phi}_{0}(s)=\frac{1}{s+\frac{\Delta_{1}}{s+\frac{\Delta_{1}}{s+\cdots}}} .
$$

Выражение (85) является представлением функции $\tilde{\phi}_{0}(s)$ следующего вида (по переменной $s)$ [63]:

$$
\tilde{\phi}_{0}(s)=\frac{-s+\sqrt{s^{2}+4 \Delta_{1}}}{2 \Delta_{1}} .
$$


Применяя обратное преобразование Лапласа к выражению (86), получаем ВКФ вида

$$
\phi_{0}(t)=\frac{1}{\Delta_{1}^{1 / 2} t} J_{1}\left(2 \Delta_{1}^{1 / 2} t\right)
$$

где $J_{1}(\cdot)$ - функция Бесселя первого рода. Такая релаксация происходит в процессах, характеризующихся затухающим гармоническим поведением. Например, выражение (87) представляет собой точный вид автокорреляционной функции скорости примесной частицы в линейной цепочке идентичных гармонических осцилляторов [11], [46], [66], где $\Delta_{1}=2 K / M$ и $K$ - коэффициент связанности соседних частиц (жесткость пружины), $M$ - масса примесной частицы. Релаксация плотности двумерного электронного газа при температуре $T=0$ и конечных волновых числах $k$ также описывается выражением (87) (см. [28]).

Рассмотренные выше примеры наглядно демонстрируют, что при известном соотношении между соответствующими релаксационными масштабами $\tau_{\nu-1}=\Delta_{\nu}^{-1 / 2}$ возможно точное определение исходной $\mathrm{BK} \Phi \phi_{0}(t)$, а также оценка особенностей ее частотного отображения $\tilde{\phi}_{0}(s)$.

4.2.3. Коэффициенты переноса. Самодиффузия и плотность вибрационных состояний в равновесной простой жидкости. Коэффициенты переноса (диффузии $D$, сдвиговой вязкости $\eta$, теплопроводности $\lambda_{T}$ ) в однокомпонентных жидкостях $\mathcal{P}=$ $\left\{D, \eta, \lambda_{T}\right\}$ связываются с корреляционными функциями потоковых переменных $A_{0}=\left\{\mathbf{v}, P_{x y}, J_{0}^{e z}\right\}$ посредством интегральных соотношений Кубо-Грина [21] следующего общего вида:

$$
\mathcal{P}=\mathcal{Q} \int_{0}^{\infty} \frac{\left\langle A_{0}(0) A_{0}(t)\right\rangle}{\left\langle A_{0}(0)^{2}\right\rangle} d t
$$

где множитель $\mathcal{Q}$ есть

$$
\mathcal{Q}=\left\{\frac{k_{\mathrm{B}} T}{m}, \frac{\left(P_{x y}\right)^{2}}{k_{\mathrm{B}} T V}, \frac{\left(J_{0}^{e z}\right)^{2}}{k_{\mathrm{B}} T^{2} V}\right\}
$$

Компоненты тензора давления определяются через вириальную формулу [67]:

$$
P_{\alpha \beta}=\sum_{i=1}^{N}\left(m v_{i \alpha} v_{i \beta}+\frac{1}{2} \sum_{i \neq j}^{N} F_{i j \alpha} r_{i j \beta}\right), \quad \alpha, \beta=x, y, z,
$$

где $F_{i j \alpha}$ обозначает $\alpha$-компоненту силы, которая возникает между частицами $i$ и $j$, находящимися на расстоянии $r_{i j}$ друг от друга. Тепловой поток вычисляется с помощью выражения

$$
J_{0}^{e z}=\sum_{i=1}^{N} v_{i z}\left(\frac{m\left|\mathbf{v}_{i}\right|^{2}}{2}+\frac{1}{2} \sum_{i \neq j}^{N} U\left(r_{i j}\right)\right)-\frac{1}{2} \sum_{i=1}^{N} \sum_{i \neq j}^{N} \mathbf{v}_{i} \mathbf{r}_{i j} \frac{\partial U\left(r_{i j}\right)}{\partial z_{i j}}
$$

где $U\left(r_{i j}\right)$ - потенциал межчастичного взаимодействия [65], [68]. 
С другой стороны, учитывая, что $\tilde{\phi}_{0}^{A A}(s)$ - лаплас-образ ВКФ

$$
\phi_{0}^{A A}(t)=\frac{\left\langle A_{0}(0) A_{0}(t)\right\rangle}{\left\langle A_{0}(0)^{2}\right\rangle},
$$

выражение (88) может быть представлено как

$$
\mathcal{P}=\mathcal{Q} \lim _{s \rightarrow 0} \tilde{\phi}_{0}^{A A}(s)
$$

где

$$
\tilde{\phi}_{0}^{A A}(s)=\frac{1}{s+\frac{\Delta_{1}^{A A}}{s+\frac{\Delta_{2}^{A A}}{s+\frac{\Delta_{3}^{A A}}{s+\cdots}}}} .
$$

В соответствии с самосогласованным подходом предположим, что на некотором $\nu$-м уровне иерархии релаксации временнь́е масштабы $\tau_{\nu-1}^{A A}=1 / \sqrt{\Delta_{\nu}^{A A}}$ выравниваются:

$$
\frac{\tau_{\nu}^{A A}}{\tau_{\nu-1}^{A A}} \rightarrow 1, \quad \nu=1,2, \ldots
$$

Тогда коэффициенты переноса будут выражаться через собственные частотные параметры:

$$
\mathcal{P}=\mathcal{Q} \times\left\{\begin{array}{l}
\frac{\Delta_{2}^{A A} \ldots \Delta_{\nu-1}^{A A}}{\Delta_{1}^{A A} \cdots \sqrt{\Delta_{\nu}^{A A}}}, \quad \nu=1,3,5, \ldots, \\
\frac{\Delta_{2}^{A A} \cdots \sqrt{\Delta_{\nu}^{A A}}}{\Delta_{1}^{A A} \ldots \Delta_{\nu-1}^{A A}}, \quad \nu=2,4,6, \ldots,
\end{array}\right.
$$

которые для каждого соответствующего процесса рассчитываются согласно общему определению (48).

Для примера рассмотрим нахождение коэффициента самодиффузии в жидкости объема $V$, где $N$ частиц взаимодействуют через сферический потенциал $U(r)$. В этом случае величина

$$
\phi_{0}^{v v}(t)=\frac{\langle\mathbf{v}(0) \cdot \mathbf{v}(t)\rangle}{\langle\mathbf{v}(0) \cdot \mathbf{v}(0)\rangle}
$$

представляет собой автокорреляционную функцию скорости частицы, а $\Delta_{j}^{v v}, j=$ $1,2, \ldots,-$ соответствующие ей частотные параметры. Спектральная плотность автокорреляционной функции скорости характеризует плотность вибрационных состояний многочастичной системы $G(\omega)$,

$$
\begin{aligned}
& G(\omega)=\int e^{i \omega t} \phi_{0}^{v v}(t) d t, \\
& G(\omega) \sim \operatorname{Re} \tilde{\phi}_{0}^{v v}(s), \quad s=\sigma+i \omega .
\end{aligned}
$$

При $\nu=1$ из (95) получаем

$$
D=\frac{k_{\mathrm{B}} T}{m} \frac{1}{\sqrt{\Delta_{1}^{v v}}},
$$


где

$$
\Delta_{1}^{v v}=\frac{\langle\dot{\mathbf{v}} \cdot \dot{\mathbf{v}}\rangle}{\langle\mathbf{v} \cdot \mathbf{v}\rangle}=\frac{4 \pi n}{3} \int_{0}^{\infty} d r g(r) r^{3}\left[\frac{3}{r^{2}} \frac{\partial U(r)}{\partial r}+\frac{\partial}{\partial r}\left(\frac{\partial U(r)}{r \partial r}\right)\right],
$$

$g(r)$ - функция радиального распределения частиц. В этом случае спектральная плотность имеет вид

$$
G(\omega) \sim \frac{\left(4 \Delta_{1}^{v v}-\omega^{2}\right)^{1 / 2}}{2 \Delta_{1}^{v v}}
$$

При $\nu=2$ находим

$$
D=\frac{k_{\mathrm{B}} T}{m} \frac{\sqrt{\Delta_{2}^{v v}}}{\Delta_{1}^{v v}}
$$

где

$$
\begin{aligned}
\Delta_{2}^{v v}= & \frac{8 \pi n}{3 m} \int_{0}^{\infty} d r g(r)\left[3\left(\frac{\partial U(r)}{\partial r}\right)^{2}+\left(r \frac{\partial}{\partial r}\left(\frac{\partial U(r)}{r \partial r}\right)\right)^{2}+\frac{\partial U(r)}{\partial r} \frac{\partial}{\partial r}\left(\frac{\partial U(r)}{r \partial r}\right)\right]+ \\
& +\frac{8 \pi^{2} n^{2}}{3 m} \int_{0}^{\infty} \int_{0}^{\infty} d r d r_{1} r^{2} r_{1}^{2} \int_{-1}^{1} d \beta_{r} g_{3}\left(r, r_{1}\right)\left[\frac{3}{r r_{1}} \frac{\partial U(r)}{\partial r} \frac{\partial U\left(r_{1}\right)}{\partial r_{1}}+\right. \\
& +\frac{r}{r_{1}} \frac{\partial U\left(r_{1}\right)}{\partial r_{1}} \frac{\partial}{\partial r}\left(\frac{\partial U(r)}{r \partial r}\right)+\frac{r_{1}}{r} \frac{\partial U(r)}{\partial r} \frac{\partial}{\partial r_{1}}\left(\frac{\partial U\left(r_{1}\right)}{r_{1} \partial r_{1}}\right)+ \\
& \left.+r_{1} \frac{\partial}{\partial r_{1}}\left(\frac{\partial U\left(r_{1}\right)}{r_{1} \partial r_{1}}\right) \frac{\partial}{\partial r}\left(\frac{\partial U(r)}{r \partial r}\right) \beta_{r}^{2}\right] .
\end{aligned}
$$

Здесь $g_{3}\left(r, r_{1}\right)$ - функция распределения трех частиц, $\beta_{r}$ - косинус угла между векторами $\mathbf{r}$ и $\mathbf{r}_{1}$. Для плотности вибрационных состояний находим выражение

$$
G(\omega) \sim \frac{2 \Delta_{1} \Delta_{2} \sqrt{4 \Delta_{2}-\omega^{2}}}{\omega^{2}\left(2 \Delta_{2}-\Delta_{1}\right)^{2}+\Delta_{1}^{2}\left(4 \Delta_{2}-\omega^{2}\right)} .
$$

При $\nu=3$ получаем

$$
D=\frac{k_{\mathrm{B}} T}{m} \frac{\Delta_{2}^{v v}}{\Delta_{1}^{v v} \sqrt{\Delta_{3}^{v v}}},
$$

где частотный параметр $\Delta_{3}^{v v}$ содержит парные, трехчастичные и четырехчастичные корреляционные функции [69]:

$$
\begin{aligned}
\Delta_{3}^{v v}=\frac{4 \pi}{m^{3}} \int_{0}^{\infty} d r g(r)\left[U_{x y}(r) U_{x x}(r) U_{y y}(r)+3 k_{\mathrm{B}} T U_{x y z}^{2}(r)\right]+ \\
\quad+\frac{n^{2}}{m^{3}} \iint d \mathbf{r} d \mathbf{r}_{1} g_{3}\left(\mathbf{r}, \mathbf{r}_{1}\right)\left[3 k_{\mathrm{B}} T U_{x x y}(r) U_{x x y}\left(r_{1}\right)+\right. \\
\left.\quad+U_{x x}(r)\left(6 U_{x y}(r) U_{x y}\left(r_{1}\right)-U_{x y}(r) U_{x y}\left(\left|\mathbf{r}-\mathbf{r}_{1}\right|\right)\right)\right]+ \\
\quad+\frac{n^{3}}{m^{3}} \iiint d \mathbf{r} d \mathbf{r}_{1} d \mathbf{r}_{2}^{\prime} g_{4}\left(\mathbf{r}, \mathbf{r}_{1}, \mathbf{r}_{2}\right) U_{x x}(r) U_{x y}\left(r_{1}\right) U_{x y}\left(r_{2}^{\prime}\right), \\
U_{x y z}(r)=\frac{\partial^{3} U(r)}{\partial r_{x} \partial r_{y} \partial r_{z}} .
\end{aligned}
$$

В данном случае плотность вибрационных состояний определяется следующим выражением:

$$
G(\omega) \sim \frac{2 \Delta_{1} \Delta_{2} \Delta_{3} \sqrt{4 \Delta_{3}-\omega^{2}}}{\left[2 \Delta_{1} \Delta_{3}-\omega^{2}\left(2 \Delta_{3}-\Delta_{2}\right)\right]^{2}+\omega^{2} \Delta^{2}\left(4 \Delta_{3}-\omega^{2}\right)} .
$$


4.2.4. Флуктуации плотности в равновесных простых жидкостях. Коллективная динамика частиц жидкости может быть количественно охарактеризована экспериментально измеряемой величиной - динамическим структурным фактором $S(k, \omega)$, который представляет собой фурье-образ функции рассеяния [21], [70]-[72]:

$$
S(k, \omega)=\frac{S(k)}{2 \pi} \int_{-\infty}^{\infty} e^{-i \omega t} F(k, t) d t,
$$

где $S(k)$ - статический структурный фактор. В свою очередь, функция рассеяния $F(k, t)$ представляет собой ВКФ флуктуаций локальной плотности $N$ частиц в жидкости

$$
F(k, t)=\frac{\left\langle\delta \rho_{k}^{*}(0) \delta \rho_{k}(t)\right\rangle}{\left\langle\left|\delta \rho_{k}(0)\right|^{2}\right\rangle},
$$

где $k=|\mathbf{k}|$ - волновое число. Поэтому весьма удобно выбрать в качестве исходной динамической переменной $A_{0}$ величину [73]-[77]

$$
A_{0}=\delta \rho(k)=\frac{1}{\sqrt{N}} \sum_{j=1}^{N} e^{i \mathbf{k} \cdot \mathbf{r}_{j}}-\delta_{\mathbf{k}, 0} n,
$$

где $\mathbf{r}_{j}(t)$ - радиус-вектор, характеризующий положение $j$-й частицы в момент времени $t$. С помощью рекуррентного соотношения (53) получаем бесконечный набор ортогональных динамических переменных [64]

$$
\begin{aligned}
\mathbf{A}(k) & =\left\{A_{0}(k), A_{1}(k), \ldots, A_{j}(k), \ldots\right\}, \\
\left\langle A_{j}^{*} A_{l}\right\rangle & =\delta_{j, l}\left\langle\left|A_{j}\right|^{2}\right\rangle, \quad A_{0}(k) \equiv \delta \rho(k),
\end{aligned}
$$

с ВКФ следующего вида:

$$
\begin{aligned}
& M_{j}(k, t)=\frac{\left\langle A_{j}^{*}(k, 0) A_{j}(k, t)\right\rangle}{\left\langle\left|A_{j}(k, 0)\right|^{2}\right\rangle}, \\
& M_{0}(k, t) \equiv F(k, t),
\end{aligned}
$$

которые удовлетворяют свойствам (16)-(18).

Определим нормированные частотные моменты динамического структурного фактора:

$$
\begin{aligned}
\omega^{(2 p)}(k) & =\int_{-\infty}^{\infty} \omega^{2 p} S(k, \omega) d \omega / \int_{-\infty}^{\infty} S(k, \omega) d \omega= \\
& =\left.(-i)^{p} \frac{d^{p} F(k, t)}{d t^{p}}\right|_{t=0}, \quad p=1,2, \ldots
\end{aligned}
$$

Конечные значения принимают лишь четные моменты $(p=2,4, \ldots)$, в то время как нечетные моменты обращаются в нуль. Учитывая, что при фиксированном значении волнового числа $k$ справедливо соотношение

$$
\left.i^{(2 j)} \frac{d^{2 j} \phi(t)}{d t^{2 j}}\right|_{t=0}=\frac{\left\langle\left[\left(i \widehat{\mathcal{L}} A_{0}\right)^{j}\right]^{*}\left(i \widehat{\mathcal{L}} A_{0}\right)^{j}\right\rangle}{\left\langle\left|A_{0}\right|^{2}\right\rangle},
$$


из (53) можно получить выражения, которые связывают частотные моменты $\omega^{(2 j)}(k)$ с частотными параметрами $\Delta_{j}(k)$ :

$$
\begin{aligned}
\Delta_{1}(k)= & \omega^{(2)}(k), \\
\Delta_{2}(k)= & \frac{\omega^{(4)}(k)}{\omega^{(2)}(k)}-\omega^{(2)}(k), \\
\Delta_{3}(k)= & \frac{\omega^{(6)}(k) \omega^{(2)}(k)-\left(\omega^{(4)}(k)\right)^{2}}{\omega^{(4)}(k) \omega^{(2)}(k)-\left(\omega^{(2)}(k)\right)^{3}}, \\
\Delta_{4}(k)= & \frac{1}{\Delta_{1}(k) \Delta_{2}(k) \Delta_{3}(k)}\left\{\omega^{(8)}(k)-\Delta_{1}(k)\left[\left(\Delta_{1}(k)+\Delta_{2}(k)\right)^{3}+\right.\right. \\
& \left.\left.\quad+2 \Delta_{2}(k) \Delta_{3}(k)\left(\Delta_{1}(k)+\Delta_{2}(k)\right)+\Delta_{2}(k) \Delta_{3}^{2}(k)\right]\right\}, \\
\Delta_{5}(k)= & \frac{1}{\Delta_{1}(k) \Delta_{2}(k) \Delta_{3}(k) \Delta_{4}(k)} \times \\
& \quad \times\left\{\omega^{(10)}(k)-2 \omega^{(8)}(k)\left[\Delta_{1}(k)+\Delta_{2}(k)+\Delta_{3}(k)+\Delta_{4}(k)\right]+\right. \\
& \quad+\omega^{(6)}(k)\left[\Delta_{1}^{2}(k)+2 \Delta_{1}(k) \Delta_{2}(k)+\Delta_{2}^{2}(k)+4 \Delta_{1}(k) \Delta_{3}(k)+\right. \\
& \left.\left.\quad+2 \Delta_{2}(k) \Delta_{3}(k)+\Delta_{3}^{2}(k)+4 \Delta_{1}(k) \Delta_{4}(k)\right]\right\}+\Delta_{4}(k) .
\end{aligned}
$$

Выражения подобного вида также известны как правила сумм [51].

Из определения (48) с учетом (53) и (108) следуют выражения для частотных параметров $\Delta_{j}(k)$. Так, например, для первых трех частотных параметров $\Delta_{1}(k)$, $\Delta_{2}(k)$ и $\Delta_{3}(k)$ имеем [64]

$$
\begin{aligned}
& \Delta_{1}(k)=\frac{k_{\mathrm{B}} T}{m} \frac{k^{2}}{S(k)}, \\
& \Delta_{2}(k)=3 \frac{k_{\mathrm{B}} T}{m} k^{2}+\frac{\rho}{m} \int \nabla_{l}^{2} U(r)\left[1-e^{i \mathbf{k} \cdot \mathbf{r}}\right] g(r) d^{3} r-\Delta_{1}(k), \\
& \Delta_{3}^{2}(k)=\frac{15}{\Delta_{2}(k)}\left(\frac{k_{\mathrm{B}} T}{m} k^{2}\right)^{2}-\frac{\left[\Delta_{1}^{2}(k)+\Delta_{2}^{2}(k)\right]^{2}}{\Delta_{2}^{2}(k)}+\frac{\mathcal{U}(k)}{\Delta_{1}^{2}(k) \Delta_{2}^{2}(k)},
\end{aligned}
$$

где через $\mathcal{U}(k)$ обозначена комбинация интегральных выражений, содержащих потенциал межчастичного взаимодействия $U(r)$ и функцию распределения трех частиц $g_{3}\left(\mathbf{r}, \mathbf{r}^{\prime}\right)$. Полное выражение для $\mathcal{U}(k)$ приводится в [78]. Отметим, что помимо значительного усложнения аналитических выражений для частотных релаксационных параметров $\Delta_{j}^{2}(k)$ с ростом порядка $j$ в них также появляются функции равновесного распределения групп из $j$ частиц, т. е.

$$
\Delta_{j}(k)=\mathcal{F}\left[\Delta_{1}(k), \Delta_{2}(k), \ldots, \Delta_{j-1}(k) ; g(r), g_{3}\left(\mathbf{r}, \mathbf{r}_{1}\right), \ldots, g_{j}\left(\mathbf{r}, \mathbf{r}_{1}, \ldots, \mathbf{r}_{j-2}\right)\right]
$$

При выборе флуктуаций плотности $A_{0}(k)=\delta \rho(k)$ в качестве исходной динамической переменной (см. (108)) при условии $k \neq 0$ получаем выражение для переменной $A_{1}(k)$ :

$$
A_{1}(k)=\frac{1}{m V} \sum_{j=1}^{N}\left(m_{j} v_{j}^{\ell}\right) e^{i \mathbf{k} \cdot \mathbf{r}_{j}},
$$


где индексом $\ell$ обозначена продольная, параллельная вектору $\mathbf{k}$ компонента. Для $A_{2}(k)$ и $A_{3}(k)$ находим

$$
\begin{aligned}
& A_{2}(k)= \frac{1}{m V} \sum_{j=1}^{N}\left\{\frac{\left(m_{j} v_{j}^{\ell}\right)^{2}}{m}+i \sum_{i>j=1}^{N} \nabla_{j} u(j, i) \mathbf{k}\left[1-e^{i \mathbf{k} \cdot\left(\mathbf{r}_{i}-\mathbf{r}_{j}\right)}\right]\right\} e^{i \mathbf{k} \cdot \mathbf{r}_{j}}- \\
& \quad-\Omega_{1}^{2}(k) \frac{1}{V} \sum_{j=1}^{N} e^{i \mathbf{k} \cdot \mathbf{r}_{j}} \\
& A_{3}(k)=\frac{1}{V} \sum_{j=1}^{N} \frac{\left(\mathbf{p}_{j} \mathbf{k}\right)^{3}}{m^{3}} e^{i \mathbf{k} \cdot \mathbf{r}_{j}}+\frac{2 i}{V m^{2}} \sum_{i>j=1}^{N}\left(\mathbf{k} \nabla_{j}\right) u(i, j)\left[\left(\mathbf{p}_{j} \mathbf{k}\right) e^{i \mathbf{k} \cdot \mathbf{r}_{j}}-\left(\mathbf{p}_{i} \mathbf{k}\right) e^{i \mathbf{k} \cdot \mathbf{r}_{i}}\right]+ \\
& \quad+\sum_{i>j=1}^{N} \frac{\mathbf{p}_{j} \boldsymbol{\nabla}_{j}}{V m^{2}}\left(\mathbf{k} \nabla_{j}\right) u(j, i)\left(e^{i \mathbf{k} \cdot \mathbf{r}_{j}}-e^{i \mathbf{k} \cdot \mathbf{r}_{i}}\right)-\left[\Delta_{1}(k)+\Delta_{2}(k)\right] A_{1}(k)
\end{aligned}
$$

Динамическая переменная $A_{4}(k)$ имеет следующий вид:

$$
\begin{aligned}
& A_{4}(k)=\frac{1}{V} \sum_{j=1}^{N} \frac{\left(\mathbf{p}_{j} \mathbf{k}\right)^{4}}{m^{4}} e^{i \mathbf{k} \cdot \mathbf{r}_{j}}+\frac{3 i}{V m^{3}} \sum_{i>j=1}^{N}\left(\mathbf{k} \nabla_{j}\right) u(j, i)\left[\left(\mathbf{p}_{j} \mathbf{k}\right)^{2} e^{i \mathbf{k} \cdot \mathbf{r}_{j}}-\left(\mathbf{p}_{i} \mathbf{k}\right)^{2} e^{i \mathbf{k} \cdot \mathbf{r}_{i}}\right]+ \\
& +\frac{2}{V m^{3}} \sum_{i>j=1}^{N} \mathbf{p}_{j} \boldsymbol{\nabla}_{j}\left(\mathbf{k} \boldsymbol{\nabla}_{j}\right) u(j, i)\left[\left(\mathbf{p}_{j} \mathbf{k}\right) e^{i \mathbf{k} \cdot \mathbf{r}_{j}}-\left(\mathbf{p}_{i} \mathbf{k}\right) e^{i \mathbf{k} \cdot \mathbf{r}_{i}}\right]- \\
& -\frac{2}{V m^{2}} \sum_{i>j=1}^{N}\left(\mathbf{k} \nabla_{j}\right)^{2} u^{2}(j, i)\left(e^{i \mathbf{k} \cdot \mathbf{r}_{j}}+e^{i \mathbf{k} \cdot \mathbf{r}_{i}}\right)- \\
& -\frac{i}{V m^{3}} \sum_{i>j=1}^{N} \mathbf{p}_{j}^{2} \boldsymbol{\nabla}_{j}^{2}\left(\mathbf{k} \nabla_{j}\right) u(j, i)\left[e^{i \mathbf{k} \cdot \mathbf{r}_{j}}-e^{i \mathbf{k} \cdot \mathbf{r}_{i}}\right]+ \\
& +\frac{i}{V m^{2}} \sum_{i>j=1}^{N} \boldsymbol{\nabla}_{j} u(j, i)\left(\boldsymbol{\nabla}_{j}-\boldsymbol{\nabla}_{i}\right)\left(\mathbf{k} \boldsymbol{\nabla}_{j}\right) u(j, i)\left(e^{i \mathbf{k} \cdot \mathbf{r}_{j}}-e^{i \mathbf{k} \cdot \mathbf{r}_{i}}\right)- \\
& -\left[\Delta_{1}(k)+\Delta_{2}(k)+\Delta_{3}(k)\right] A_{2}(k)-\left[\Delta_{1}(k)+\Delta_{2}(k)\right] \Delta_{1}^{2}(k) A_{0}(k) .
\end{aligned}
$$

Из выражений (108), (116)-(119) видно, что при выборе в качестве исходной динамической переменной флуктуации локальной плотности $A_{0}(k)$ функция $M_{1}(k, t)$ представляет собой ВКФ флуктуаций продольной компоненты импульса, $M_{2}(k, t)$ связывается непосредственно с ВКФ флуктуаций энергии и т. д. Следовательно, в пределе малых волновых чисел $(k \rightarrow 0)$ величины $M_{0}(k, t), M_{1}(k, t)$ и $M_{2}(k, t)$ могут быть сопоставлены с автокорреляторами, соответствующими трем сохраняющимся гидродинамическим переменным. Динамические переменные высоких порядков $\left(A_{j}\right.$ при $\left.j \geqslant 3\right)$ описывают релаксацию более "сложных" процессов, содержащих кросс-корреляции импульсов, энергий, потоков и т. д. Так, например, процесс, характеризующийся динамической переменной $A_{3}(k)$, отождествляется с флуктуациями продольного потока энергии [47]. 
Сокращение описания может быть реализовано в рамках постулата о выравнивании временнь́х масштабов

$$
\frac{\tau_{j}}{\tau_{j-1}} \rightarrow 1, \quad j=1,2, \ldots
$$

В самом простом приближении, согласующемся с положениями гидродинамической теории, полагаем, что времена релаксации последующих ВКФ по сравнению с масштабами первых трех динамических переменных $A_{0}, A_{1}$ и $A_{2}$ являются сопоставимыми; $\tau_{3}(k) \simeq \tau_{4}(k) \simeq \tau_{j}(k), j>3$, что есть

$$
\Delta_{4}(k)=\Delta_{5}(k)=\Delta_{6}(k)=\cdots=\Delta_{j}(k), \quad j \geqslant 4 .
$$

Тогда из (62) по аналогии с (85)-(87) получаем

$$
\widetilde{M}_{3}(s)=\frac{-s+\sqrt{s^{2}+4 \Delta_{4}}}{2 \Delta_{4}}
$$

И

$$
M_{3}(t)=\frac{1}{\Delta_{4}^{1 / 2} t} J_{1}\left(2 \Delta_{4}^{1 / 2} t\right) .
$$

Подставляя (121) в (62), с учетом (106) находим выражение для динамического структурного фактора:

$$
\begin{aligned}
& S(k, \omega)=\frac{S(k)}{2 \pi} \Delta_{1}(k) \Delta_{2}(k) \Delta_{3}(k)\left[4 \Delta_{4}(k)-\omega^{2}\right]^{1 / 2}\left\{\Delta_{1}^{2}(k) \Delta_{3}^{2}(k)+\right. \\
&+\omega^{2}\left[\Delta_{1}^{2}(k) \Delta_{4}(k)-2 \Delta_{1}(k) \Delta_{3}^{2}(k)-\Delta_{1}^{2}(k) \Delta_{3}(k)+\right. \\
&\left.+2 \Delta_{1}(k) \Delta_{2}(k) \Delta_{4}(k)-\Delta_{1}(k) \Delta_{2}(k) \Delta_{3}(k)+\Delta_{2}^{2}(k) \Delta_{4}(k)\right]+ \\
&+\omega^{4}\left[\Delta_{3}^{2}(k)-2 \Delta_{1}(k) \Delta_{4}(k)+2 \Delta_{1}(k) \Delta_{3}(k)-2 \Delta_{2}(k) \Delta_{4}(k)+\right. \\
&\left.\left.+\Delta_{2}(k) \Delta_{3}(k)\right]+\omega^{6}\left[\Delta_{4}(k)-\Delta_{3}(k)\right]\right\}^{-1} .
\end{aligned}
$$

Как видно из (123), величина $S(k, \omega)$ в области конечных значений $k$, соответствующих микроскопическим пространственным масштабам, полностью определяется первыми четырьмя частотными параметрами, которые содержат информацию о микроскопических свойствах системы и выражаются через потенциал межчастичного взаимодействия, а также двух-, трех-, четырехчастичными функциями распределения [47], [79], [80].

Как известно, в спектрах динамического структурного фактора, измеряемых в опытах по неупругому рассеянию нейтронов и рентгеновских лучей в простых жидкостях, помимо упругой компоненты (на нулевой частоте) также присутствуют два симметричных неупругих пика, расположенных на частотах $\pm \omega_{\mathrm{c}} \neq 0$ (см., например, [81]). Хотя полная форма спектров $S(k, \omega)$ при конечных значениях волнового числа в целом напоминает форму известного гидродинамического триплета, наблюдаемого в опытах по рассеянию света, в отличие от гидродинамического случая, пики здесь не являются отчетливо разделенными [82]-[87]. Неупругие особенности спектров рассеяния проявляются в ВКФ продольного потока [88]

$$
G_{J}(k, t)=\frac{\left\langle J^{\mathrm{L}}(k, 0) J^{\mathrm{L}}(k, t)\right\rangle}{\left\langle\left|J^{\mathrm{L}}(k, 0)^{2}\right|\right\rangle},
$$


которая связана с динамическим структурным фактором следующим соотношением:

$$
S(k) \Delta_{1}(k) \widetilde{G}_{J}(k, \omega)=\omega^{2} S(k, \omega) .
$$

В свою очередь, соотношение (125) может быть получено из равенства

$$
\Delta_{1}(k) G_{J}(k, t)=-\frac{\partial^{2} F(k, t)}{\partial t^{2}} .
$$

В случае однокомпонентных простых жидкостей величина $\widetilde{G}_{J}(k, \omega)$ характеризуется минимумом, располагающимся на нулевой частоте $(\omega=0)$, и двумя высокочастотными (при $\omega \neq 0$ ) максимумами. Положение и ширина максимумов в спектре $\widetilde{G}_{J}(k, \omega)$ определяются решением так называемого уравнения дисперсии

$$
s+\frac{\Delta_{1}(k)}{s}+\Delta_{2}(k) \widetilde{M}_{2}(k, s)=0
$$

относительно $s=s(k)$, где выражение для $\widetilde{M}_{2}(k, s)$ следует из $(121)$ и имеет вид

$$
\widetilde{M}_{2}(k, s)=\frac{2 \Delta_{4}(k)}{s\left[2 \Delta_{4}(k)-\Delta_{3}(k)\right]+\Delta_{3}(k) \sqrt{s^{2}+4 \Delta_{4}(k)}} .
$$

В общем виде уравнение (127) имеет комплексные решения $s=\operatorname{Re}[s(k)]+i \operatorname{Im}[s(k)]$, где $\operatorname{Im}[s(k)]$ определяет положение неупругих пиков в $\widetilde{G}_{J}(k, \omega)$, a $\operatorname{Re}[s(k)]$ характеризует ширину этих пиков.

Введем следующие безразмерные величины:

$$
\mathcal{Q}(k)=2 \frac{\Delta_{4}(k)}{\Delta_{3}(k)}-1, \quad \xi(k)=\frac{s^{2}}{\Delta_{4}(k)} .
$$

Тогда условие существования высокочастотных пиков в уравнении (127) может быть записано в виде

$$
s^{2}+s \Delta_{2}(k) \frac{1+\mathcal{Q}(k)}{s \mathcal{Q}(k)+\sqrt{s^{2}+4 \Delta_{4}(k)}}+\Delta_{1}(k)=0 .
$$

Для анализа этого уравнения рассмотрим следующие предельные ситуации.

1. Область перехода к гидродинамическому пределу удовлетворяет условию $|\xi(k)| \ll 1$, которое позволяет охватить область условно малых частот (больших временны́х масштабов). Тогда уравнение дисперсии принимает следующий вид:

$$
s^{3}+\frac{2 \Delta_{4}^{1 / 2}(k)}{\mathcal{Q}(k)} s^{2}+\left[\Delta_{1}(k)+\frac{\Delta_{2}(k)(1+\mathcal{Q}(k))}{\mathcal{Q}(k)}\right] s+\frac{2 \Delta_{4}^{1 / 2}(k) \Delta_{1}(k)}{\mathcal{Q}(k)}=0 .
$$

Решая данное уравнение в соответствии со схемой Маунтейна [89], получаем приближенные решения вида

$$
s_{1,2}(k)= \pm i c_{s} k-\Gamma k^{2}, \quad s_{3}(k)=-D_{T} k^{2},
$$


где адиабатической скорости звука $c_{\mathrm{s}}$, коэффициенту затухания звука $\Gamma$, температуропроводности $D_{T}$ и отношению удельных теплоемкостей $\gamma=C_{p} / C_{v}$ сопоставляются выражения

$$
\begin{gathered}
c_{\mathrm{S}}=\sqrt{\gamma} c_{0}, \quad \Delta_{1}(k)=c_{0}^{2} k^{2}, \\
1+\frac{\Delta_{2}(k)[1+\mathcal{Q}(k)]}{\Delta_{1}(k) \mathcal{Q}(k)}=\gamma, \\
\Delta_{1}(k)+\frac{\Delta_{2}(k)(1+\mathcal{Q}(k))}{\mathcal{Q}(k)}=c_{\mathrm{s}}^{2} k^{2}, \\
\frac{\gamma-1}{\gamma} \frac{\Delta_{4}^{1 / 2}(k)}{\mathcal{Q}(k)}=\Gamma k^{2}, \\
2 \frac{\Delta_{4}^{1 / 2}(k)}{\gamma \mathcal{Q}(k)}=D_{T} k^{2},
\end{gathered}
$$

$c_{0}$ - изотермическая скорость звука. Выражения (133) в пределе малых значений волнового числа $k$ соответствуют результатам гидродинамической теории Ландау-Плачека [90]. Действительная и мнимая части первых двух решений в (132) определяют положение и ширину дублета Мандельштама-Бриллюэна ${ }^{4)}$ (см. рис. 1).

2. Рассмотрим высокочастотную область (независимо от значения волнового числа $k$ ), определяемую условием $s^{2} / \Delta_{4}(k) \gg 1$. В этом случае уравнение дисперсии имеет решения

$$
s_{1,2}(k) \approx \pm i \sqrt{\Delta_{1}(k)+\Delta_{2}(k)} \equiv \pm i \omega_{L}(k),
$$

которые воспроизводят обычный “мгновенный” твердотельный отклик [50].

Как видно из уравнения дисперсии (127) и получаемых решений (133)-(137), ширина и положение боковых пиков в спектрах полностью определяются четырьмя частотными параметрами $\Delta_{j}(k), j=1,2,3,4$. Это непосредственно указывает на то, что высокочастотные спектральные особенности на микроскопических пространственных масштабах определяются двух-, трех- и четырехчастичными корреляциями. Несмотря на то что высокочастотная динамика может рассматриваться как проявление твердотельных свойств, достаточно большие времена жизни высокочастотных возбуждений (по сравнению с твердотельными) суть характерная особенность динамики жидкости, где двух-, трех- и четырехчастичные корреляции выражены на пространственных масштабах, сопоставимых с атомно-молекулярными размерами [88].

4.3. Масштабируемость в релаксации. В случае, когда релаксация различных процессов характеризуется масштабируемостью, выражаемой соотношением вида (72), соответствующее теоретическое описание может быть реализовано в рамках представленного здесь самосогласованного подхода. Теоретические модели, развиваемые в рамках приближений взаимодействующих (связанных) мод, непосредственно относятся к данному классу.

4) Отметим, что приближенные решения (133) являются справедливыми, когда отношение между $\Delta_{4}(k)$ и $\Delta_{3}(k)$ значительно по сравнению с отношениями между $\Delta_{1}(k), \Delta_{2}(k)$ и $\Delta_{3}(k)$. Это условие связано с расхождением значений частотных параметров в гидродинамическом пределе, что позволяет перейти к процедуре Маунтейна [89]. 


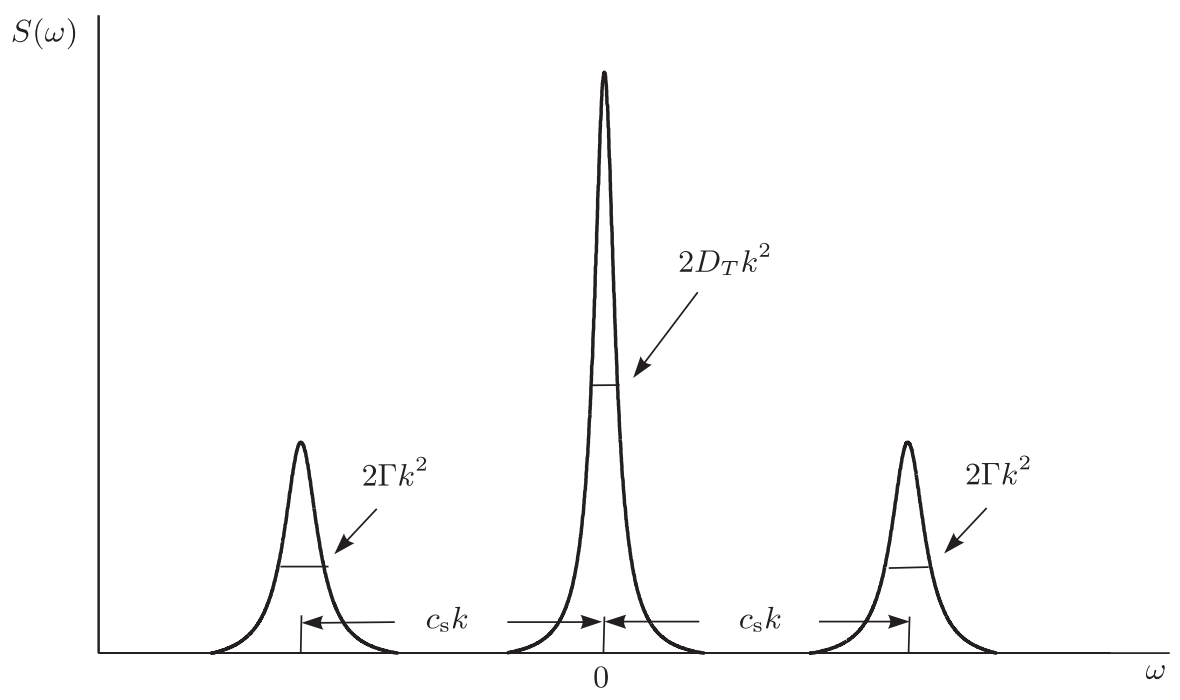

a

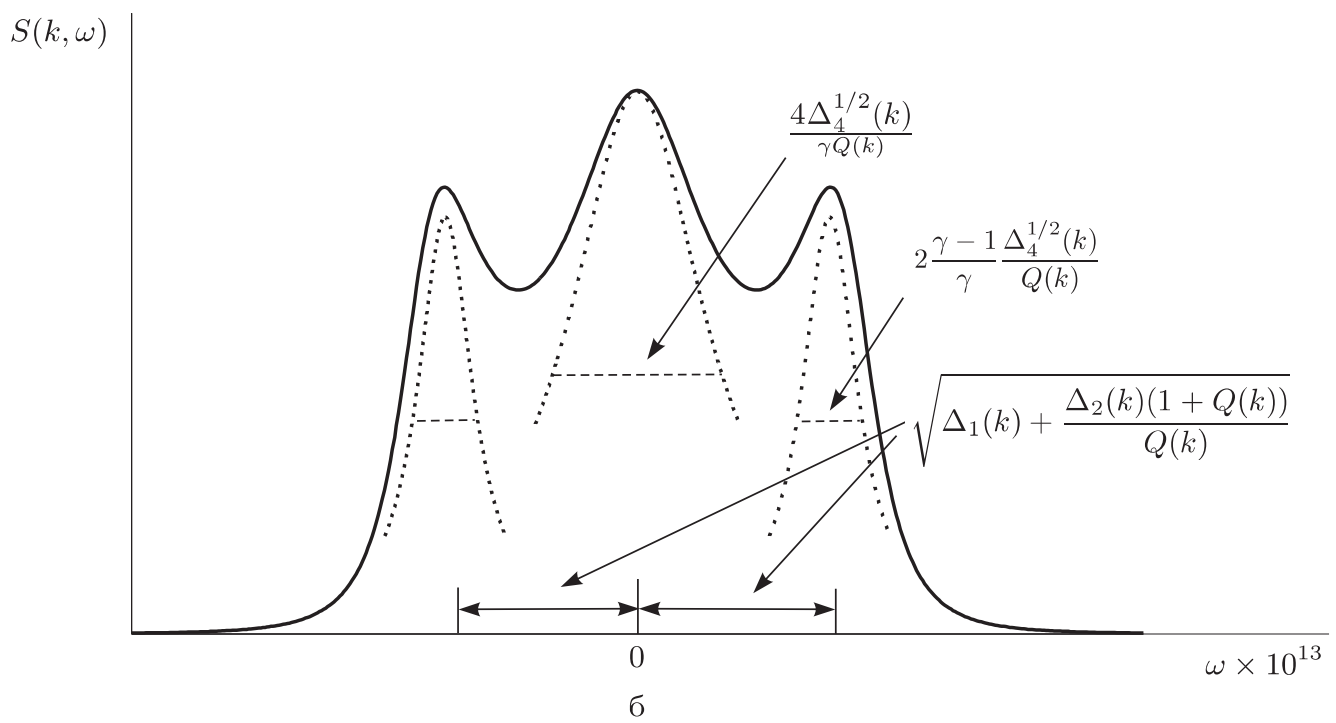

Рис. 1. а - Типичный спектр динамического структурного фактора, измеряемый в опытах по рассеянию света в простой жидкости [91], [92], который соответствует гидродинамической области с малыми значениями $k$. б - Спектр динамического структурного фактора, измеряемый в опытах по неупругому рассеянию медленных нейтронов и рентгеновских лучей [70], [71], который соответствует микроскопическим пространственным масштабам с конечными значениями $k$. Положения и ширины боковых пиков определяются выражениями (135) и (136) соответственно. Полуширина центрального пика определяется выражением (137). 
Так, например, при выполнении скейлингового соотношения в релаксации процессов, соотносимых с переменными $A_{\nu}$ и $A_{\nu+1}$ набора $\mathbf{A}$,

$$
\phi_{\nu+1}(t)=\sum_{i} \mathcal{A}_{i} \phi_{\nu}(t)^{p_{i}}, \quad \sum_{i} \mathcal{A}_{i}=1, \quad \nu \geqslant 0, \quad p_{i}>0
$$

$\nu$-е уравнение цепочки (49) принимает вид

$$
\frac{d \phi_{\nu}(t)}{d t}=\Delta_{\nu+1} \int_{0}^{\infty} \phi_{\nu}(t-\tau)\left[\sum_{i} \mathcal{A}_{i} \phi_{\nu}(t)^{p_{i}}\right] d \tau
$$

Уравнение вида (140) может быть решено численно при начальных условиях (ср. с (16)-(18))

$$
\left.\phi_{\nu}(t)\right|_{t=0}=1,\left.\quad \frac{d \phi_{\nu}(t)}{d t}\right|_{t=0}=0 .
$$

4.3.1. Структурная релаксация в переохлажденных жидкостях. Приближения взаимодействующих мод. Выберем в качестве исходной динамической переменной величину

$$
A_{0}(t) \equiv \rho_{\mathbf{k}}^{(s)}(t)=e^{i \mathbf{k} \cdot \mathbf{r}_{s}(t)},
$$

которая представляет собой фурье-компоненту от $\rho_{s}(\mathbf{r}, t)=\delta\left[\mathbf{r}-\mathbf{r}_{s}(t)\right]($ см. выражение (2)) и ВКФ которой представляет собой так называемую функцию рассеяния [21]

$$
\phi_{0}(k, t) \equiv F_{s}(k, t)=\left\langle e^{i \mathbf{k}\left(\mathbf{r}_{s}(t)-\mathbf{r}_{s}(0)\right)}\right\rangle .
$$

Здесь

$$
S_{s}(k, \omega)=\frac{1}{2 \pi} \int_{-\infty}^{\infty} e^{-i \omega t} F_{s}(k, t) d t
$$

- некогерентная составляющая неупругого рассеяния нейтронов, содержащая информацию об одночастичной динамике.

Запишем первые два уравнения цепочки (49):

$$
\begin{aligned}
& \frac{d F_{s}(k, t)}{d t}=-\Delta_{1}(k) \int_{0}^{\infty} F_{s}(k, t-\tau) \phi_{1}^{(s)}(k, \tau) d \tau \\
& \frac{d \phi_{1}(k, t)}{d t}=-\Delta_{2}(k) \int_{0}^{\infty} \phi_{1}^{(s)}(k, t-\tau) \phi_{2}^{(s)}(k, \tau) d \tau,
\end{aligned}
$$

где частотный параметр $\Delta_{1}(k)=k_{\mathrm{B}} T k^{2} / m$ в данном случае характеризует тепловое движение частицы,

$$
\Delta_{2}(k)=2 \Delta_{1}(k)+\frac{\left\langle\nabla^{2} U\left(\mathbf{r}_{s}\right)\right\rangle}{3 m}+\mathcal{O}\left(k^{2}\right), \quad\left|\mathbf{r}_{s}\right| \simeq \frac{2 \pi}{k},
$$

функция памяти первого порядка $\phi_{1}^{(s)}(k, t)$ отождествляется с ВКФ продольной компоненты скорости частицы, а функция памяти $\phi_{2}^{(s)}(k, t)-\operatorname{BK\Phi ~силы~} \mathbf{f}_{s}$, действующей на частицу со стороны окружения. Объединяя (145) и (146), получаем обобщенное уравнение Ланжевена в виде [61]

$$
\frac{d^{2} F_{s}(k, t)}{d t^{2}}+\Delta_{1}(k) F_{s}(k, t)+\Delta_{2}(k) \int_{0}^{t} d \tau \phi_{2}^{(s)}(k, t-\tau) \frac{d F_{s}(k, \tau)}{d \tau}=0
$$


для решения которого необходимо знать поведение функции памяти $\phi_{2}^{(s)}(k, t)$. Следуя ключевой идее приближений взаимодействующих мод о коррелируемости процессов [59], связанных со стохастической силой $f_{s}$ и структурной релаксацией непосредственно, можно записать скейлинговое выражение

$$
\begin{aligned}
\phi_{2}^{(s)}(k, t) & =\mathcal{A}_{1} F_{s}(k, t)+\mathcal{A}_{2} F_{s}(k, t)^{p}, \\
0 & \leqslant \mathcal{A}_{1}, \mathcal{A}_{2} \leqslant 1, \quad \mathcal{A}_{1}+\mathcal{A}_{2}=1, \quad p>1,
\end{aligned}
$$

где $\mathcal{A}_{1}, \mathcal{A}_{2}$ - весовые коэффициенты. Подставляя выражение (149) в (148), получаем уравнение, которое может быть решено численно и дает широкий набор решений, способных воспроизводить особенности некогерентной функции рассеяния $F_{s}(k, t)$ в области перехода "переохлажденная жидкость - стекло". На рис. 2 в качестве примера показаны решения для $F_{s}(k, t)$, получаемые из (148) в двух частных случаях:

а) когда выражение (149) имеет вид

$$
\phi_{2}^{(s)}(k, t)=F_{s}(k, t)^{2},
$$

что представляет собой упрощенную модель Левгезера [29], [93] (см. рис. 2а);

б) когда выражение (149) может быть представлено в виде

$$
\phi_{2}^{(s)}(k, t)=\mathcal{A}_{1} F_{s}(k, t)+\mathcal{A}_{2} F_{s}(k, t)^{2},
$$

что есть упрощенная $M_{12}$-модель Гётце-Сьегрена [94] (см. рис. 2б).

Как видно из рис. 2, обе модели воспроизводят переход системы из эргодической в неэргодическую фазу, связываемый с появлением плато во временно́й зависимости $F_{s}(k, t)$. При этом переходе функция рассеяния начинает характеризоваться двухступенчатой релаксацией, для которой быстрые релаксационные процессы, связываемые преимущественно с колебательной динамикой частиц, обозначаются как $\beta$-релаксация, в то время как медленные процессы, ответственные за структурные трансформации, ассоциируются с $\alpha$-релаксацией [21]. Здесь переход в неэргодическую фазу определяется частотными параметрами $\Delta_{1}(k)$ и $\Delta_{2}(k)$. Так, на примере модели (150) для характеризации перехода удобно ввести параметр $\lambda=$ $\Delta_{2}(k) / 4 \Delta_{1}(k)$. Тогда появление перехода связывается с условием $\lambda=1$ (см. рис. $\left.2 \mathrm{a}\right)$.

Важной особенностью приближений взаимодействующих мод является то, что они позволяют воспроизводить характерные этапы во временно́м поведении ВКФ для переохлажденной жидкости. Так, поведение $F_{s}(k, t)$ на начальном этапе $\beta$-релаксации характеризуется следующей зависимостью:

$$
F_{s}(k, t) \simeq 1-\frac{\Delta_{1}(k)}{2} t^{2}+\mathcal{O}\left(t^{4}\right)
$$

что следует из (21). Далее, завершающий этап в $\beta$-релаксации, предшествующий появлению плато в $F_{s}(k, t)$ (см. рис. 2a), описывается так называемым критическим законом

$$
F_{s}(k, t) \simeq f^{(s)}(k)+h(k)\left(\frac{t}{\tau_{0}(k)}\right)^{-a}
$$



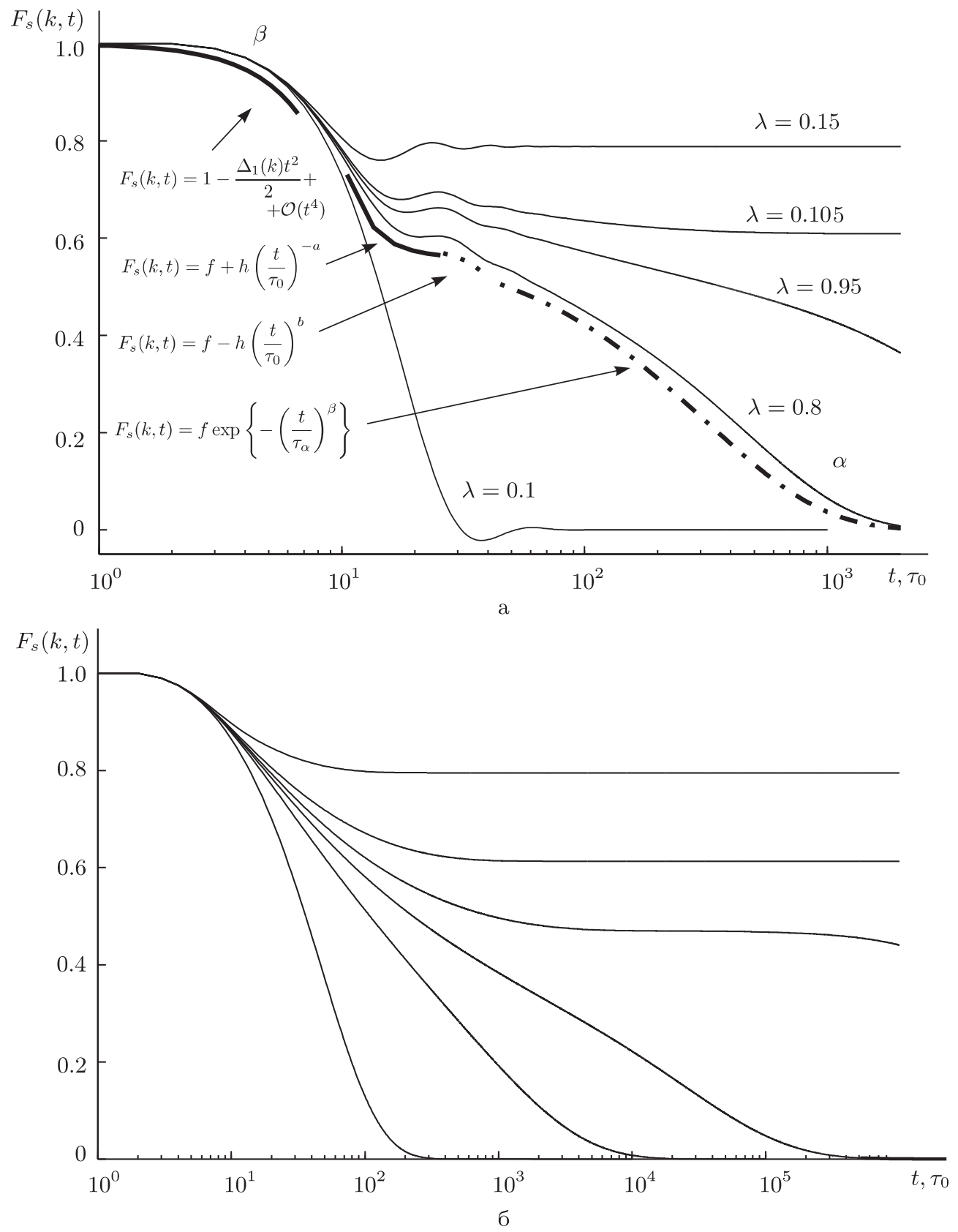

Рис. 2. Функция рассеяния $F_{s}(k, t)$ получаемая из численного решения уравнения (148) в двух различных приближениях вида (149): результаты, получающиеся в случае приближения (150) (а) и в случае приближения (151) (б). Здесь $\lambda=\Delta_{2}(k) / 4 \Delta_{1}(k), \tau_{0}$ - единичный временно́й масштаб. 
где $f^{(s)}(k)$ - параметр неэргодичности, характеризующий высоту плато в функции $F_{s}(k, t), \tau_{0}(k)$ - релаксационный масштаб. В соответствии с приближением (150) показатель степени в критическом законе определяется из следующего выражения:

$$
\frac{\Gamma^{2}(1-a)}{\Gamma(1-2 a)}=\frac{\Delta_{2}(k)}{\Delta_{1}(k)}\left[1-f^{(s)}(k)\right]^{3},
$$

где $\Gamma(\cdot)$ - гамма-функция. "Начальный” этап $\alpha$-релаксации (см. рис. 2б), характеризующий последующее затухание ВКФ после устойчивого поведения с $F_{s}(k, t) \simeq$ $f^{(s)}(k)$, происходит в соответствии с релаксацией фон Швейдлера [94]:

$$
F_{s}(k, t) \simeq f^{(s)}(k)-h(k)\left(\frac{t}{\tau_{0}(k)}\right)^{b},
$$

где в случае приближения (150) имеем связь показателя степени $b$ с частотными параметрами:

$$
\frac{\Gamma^{2}(b+1)}{\Gamma(2 b+1)}=\frac{\Delta_{2}(k)}{\Delta_{1}(k)}\left[1-f^{(s)}(k)\right]^{3} .
$$

Наконец, следует также отметить, что временно́е поведение ВKФ $F_{s}(k, t)$ на больших временнь́х масштабах, соответствующих масштабам структурной релаксации, получаемое из (149), позволяет приближенно воспроизводить релаксационное поведение, описываемое растянутой экспонентой (функцией Кольрауша):

$$
F_{s}(k, t) \simeq f^{(s)}(k) e^{-\left(t / \tau_{\alpha}\right)^{\beta}}
$$

где $\beta<1$, а $\tau_{\alpha}$ отождествляется с временем структурной релаксации.

Благодарности. Исследование выполнено за счет гранта Российского научного фонда (проект № 14-12-01185). Автор выражает признательность Р. М. Хуснутдинову (КФУ, г. Казань) за полезные замечания и помощь в выполнении численных расчетов, результаты которых представлены на рис. 2 ; А.Г. Новикову (ФЭИ им. А. И. Лейпунского, г. Обнинск), Р. Hänggi (Universität Augsburg, Augsburg), J.-L. Barrat (Université Joseph Fourier, Grenoble), M. H. Lee (University of Georgia, Athens) за многочисленные обсуждения вопросов, которые затрагиваются в настоящей работе. Автор выражает искреннюю признательность В.Н. Рыжову и В. В. Бражкину за полезные вопросы, советы, а также обсуждения результатов данной работы на семинарах, проходивших в ИФВД РАН в 2013-2014 гг.

\section{Список литературы}

[1] Д. Н. Зубарев, УФН, 71:1 (1960), 71-116.

[2] Д. Н. Зубарев, Ю. Г. Рудой, УФН, 163:3 (1993), 103-106.

[3] З. З. Боголюбов, С. В. Тябликов, Докл. АН СССР, 126 (1959), 53-56.

[4] Ю. А. Церковников, Докл. АН СССР, 143 (1962), 832-835.

[5] Ю. А. Церковников, ТМФ, 49:2 (1981), 219-233.

[6] Ю. Г. Рудой, Ю. А. Церковников, ТМФ, 14:1 (1973), 102-122.

[7] А. А. Владимиров, Д. Иле, Н. М. Плакида, ТМФ, 145:2 (2005), 240-255.

[8] Ю. А. Церковников, ТМФ, 154:1 (2008), 197-206. 
[9] Н. М. Плакида, ТМФ, 5:1 (1970), 147-153.

[10] H. B. Callen, I. A. Welton, Phys. Rev., 83:1 (1951), 34-40.

[11] R. Zwanzig, Nonequilibrium Statistical Mechanics, Oxford Univ. Press, Oxford, 2004.

[12] Д. Н. Зубарев, В. Г. Морозов, Г. Рёпке, Статистическая механика неравновесных процессов, т. 2, Физматлит, М., 2002.

[13] R. Kubo, J. Phys. Soc. Japan, 12:6 (1957), 570-586.

[14] U. Balucani, M. H. Lee, V. Tognetti, Phys. Rep., 373:6 (2003), 409-492.

[15] И. К. Камилов, А. К. Муртазаев, Х. К. Алиев, УФН, 169:7 (1999), 773-795.

[16] И. М. Лифшиц, С.А. Гредескул, Л.А. Пастур, Введение в теорию неупорядоченных систем, Наука, М., 1982.

[17] Б. А. Клумов, УФН, 180:10 (2010), 1095-1108.

[18] B. J. Berne, G. D. Harp, "On the calculation of time correlation functions", Advances in Chemical Physics, 17, eds. I. Prigogine, S. A. Rice, Wiley, New York, 1970, 63-227.

[19] J. R. D. Copley, S. W. Lovesey, Rep. Prog. Phys., 38:4 (1975), 461-563.

[20] K. Tankeshwar, G. S. Dubey, K. Pathak, J. Phys. C, 21:22 (1988), L811-L814.

[21] J. P. Hansen, I. R. McDonald, Theory of Simple Liquids, Academic Press, London, 2006.

[22] I. M. de Schepper, E. G. D. Cohen, C. Bruin, J. C. van Rijs, W. Montfrooij, L. A. de Graaf, Phys. Rev. A, 38:1 (1988), 271-287.

[23] А. И. Олемской, И. В. Коплык, УФН, 165:10 (1995), 1105-1144.

[24] А. И. Олемской, УФН, 166:7 (1996), 697-715.

[25] M. H. Lee, Phys. Rev. Lett., 49:15 (1982), 1072-1075.

[26] M. H. Lee, Phys. Rev. E, 62:2 (2000), 1769-1772.

[27] M. H. Lee, Phys. Rev. B, 26:5 (1983), 2547-2551.

[28] M. H. Lee, J. Hong, J. Florencio, Jr., Phys. Scr., T19B (1987), 498-504.

[29] E. Leutheusser, Phys. Rev. A, 29:5 (1984), 2765-2773.

[30] W. Gotze, L. Sjogren, Rep. Prog. Phys., 55:3 (1992), 241-376.

[31] В. Ю. Шурыгин, Р. М. Юльметьев, ТМФ, 83:2 (1990), 222-235.

[32] R. M. Yul'met'yev, Acta Phys. Polon. A, 58:6 (1980), 801-810.

[33] R. M. Yul'met'yev, Acta Phys. Polon. A, 65:1 (1984), 33-38.

[34] Р. М. Юльметьев, ТМФ, 30:2 (1977), 264-281.

[35] Ю.К. Товбин, Молекулярная теория адсорбиии в пористых телах, Физматлит, М., 2012.

[36] В.Н. Рыжов, А.Ф. Барабанов, М. В. Магницкая, Е.Е. Тареева, УФН, 178:10 (2008), 1118-1124.

[37] V. V. Brazhkin, Yu. D. Fomin, A. G. Lyapin, V. N. Ryzhov, K. Trachenko, Phys. Rev. E., 85:3 (2012), 031203, 12 pp.

[38] V. V. Brazhkin, Yu. D. Fomin, A. G. Lyapin, V. N. Ryzhov, E. N. Tsiok, K. Trachenko, Phys. Rev. Lett., 111:14 (2013), 145901, 5 pp.

[39] Я.И. Френкель, Кинетическая теория жидкостей, Наука, Л., 1975.

[40] N.H. March, Liquid Metals. Concepts and Theory, Cambridge Univ. Press, Cambridge, 1990.

[41] P. J. Steinhardt, D. R. Nelson, M. Ronchetti, Phys. Rev. B, 28:2 (1983), 784-805.

[42] Р. С. Берри, Б. М. Смирнов, УФН, 179:2 (2009), 147-177.

[43] М. Рид, Б. Саймон, Методы современной математической физики, т. 1: Функциональный анализ, Мир, М., 1977.

[44] R. Zwanzig, Phys. Rev., 124:4 (1961), 983-992.

[45] H. Mori, Prog. Theor. Phys., 33:3 (1965), 423-455; 34:3, 399-416.

[46] A. V. Mokshin, R. M. Yulmetyev, P. Hänggi, Phys. Rev. Lett., 95:20 (2005), 200601-200601, 4 pp., arXiv: cond-mat/0511308. 
[47] А.В. Мокшин, Р. М. Юльметьев, Микроскопическая динамика простых жидкостей, Центр инновационных технологий, Казань, 2006.

[48] Н. Н. Боголюбов, Проблемы динамической теории в статистической физике, Гостехиздат, М.-Л., 1946.

[49] Н.Н. Боголюбов (ред.), Статистическая физика и квантовая теория поля, Наука, M., 1973.

[50] U. Balucani, M. Zoppi, Dynamics of the Liquid State, Clarendon Press, Oxford, 1994.

[51] Г. Темперли, Дж. Роулинсон, Дж. Рашбрук (ред.), Физика простых жидкостей. Экспериментальные исследования, Мир, М., 1973.

[52] A. V. Mokshin, R. M. Yulmetyev, P. Hänggi, New J. Phys., 7:1 (2005), 9, 10 pp.

[53] R. R. Nigmatullin, Physica A: Statist. Mech. Appl., 363:2 (2006), 282-298.

[54] R. R. Nigmatullin, Physica A: Statist. Mech. Appl., 285:3-4 (2000), 547-565.

[55] A. Wierling, Europ. Phys. J. B, 85 (2012), 20, 9 pp.

[56] A. Wierling, I. Sawada, Contributions to Plasma Physics, 52:1 (2012), 49-52.

[57] В.Н. Рыжов, Е.Е. Тареева, Т. И. Щелкачева, Н. М. Щелкачев, ТМФ, 141:1 (2004), 131-140.

[58] R.E. Ryltsev, N. M. Chtchelkatchev, V. N. Ryzhov, Phys. Rev. E, 110:2 (2013), 025701, 5 pp., arXiv: 1301.2162.

[59] K. Kawasaki, Ann. Phys., 61 (1970), 1-56.

[60] A. V. Mokshin, R. M. Yulmetyev, R. M. Khusnutdinov, P. Hänggi, ФTT, 48:9 (2006), $1662-1665$.

[61] А. В. Мокшин, А. В. Чванова, Р. М. Хуснутдинов, ТМФ, 171:1 (2012), 135-149.

[62] J. Florencio, Jr., M. H. Lee, Phys. Rev. A, 31:5 (1985), 3231-3236.

[63] М. Абрамовиц, И. Стиган (ред.), Справочник по специалъным функииям с формулами, графиками и математическими таблицами, Наука, М., 1979.

[64] А.В. Мокшин, Р. М. Юльметьев, Р. М. Хуснутдинов, П. Хангги, ЖЭТФ, 130:6(12) (2006), 974-983.

[65] A. V. Mokshin, Discontin. Nonlinearity Complex., 2:1 (2013), 43-56.

[66] R. J. Rubin, Phys. Rev., 131:3 (1963), 964-989.

[67] D. J. Evans, G. Morriss, Statistical Mechanics of Nonequilibrium Liquids, Cambridge Univ. Press, New York, 2008.

[68] А. В. Мокшин, С. О. Забегаев, Р. М. Хуснутдинов, ФTТ, 53:3 (2011), 532-537.

[69] R. Bansal, K. N. Pathak, Phys. Rev. A, 9:6 (1974), 2773-2782.

[70] N. M. Blagoveshchenskii, A. G. Novikov, V. V. Savostin, Crystallography Reports, 56:7 (2011), 1096-1099.

[71] N. M. Blagoveshchenskii, A. G. Novikov, Physica B: Condens. Matter, 406:9 (2011), 1749-1751.

[72] N. M. Blagoveshchenskii, A. G. Novikov, V.V. Savostin, Physica B: Condens. Matter, 407:23 (2012), 4567-4569.

[73] R. M. Yulmetyev, A. V. Mokshin, P. Hänggi, V. Yu. Shurygin, Phys. Rev. E, 64:5 (2001), 057101, 4 pp., arXiv: cond-mat/0111467.

[74] R. M. Yulmetyev, A. V. Mokshin, T. Scopigno P. Hänggi, J. Phys.: Condens. Matter, 15:14 (2003), 2235-2257.

[75] R. M. Yulmetyev, A.V. Mokshin, P. Hänggi, V. Yu. Shurygin, Писъма в ЖЖЭТФ, 76:3 (2002), 181-184.

[76] R. M. Yulmetyev, A. V. Mokshin, P. Hänggi, Phys. Rev. E, 68:5 (2003), 051201-051201, 5 pp., arXiv: cond-mat/0401314.

[77] A. V. Mokshin, R. M. Yulmetyev, P. Hänggi, J. Chem. Phys., 121:15 (2004), 7341-7346, arXiv: cond-mat/0506636. 
[78] E. Michler, H. Hahn, P. Schofield, J. Phys. F: Metal Phys., 7:5 (1977), 869-875.

[79] Ю. М. Гуфан, И. Н. Мощенко, ФТТ, 33:4 (1991), 1166-1172.

[80] И. А. Осипенко, О.В. Кукин, А.Ю. Гуфан, Ю.М. Гуфан, ФТT, 55:12 (2013), 2289-2296.

[81] T. Scopigno, G. Ruocco, F. Sette, Rev. Modern Phys., 77:3 (2005), 881-933, arXiv: cond-mat/0503677.

[82] Б. Б. Маркив, И. П. Омелян, М. В. Токарчук, ТМФ, 154:1 (2008), 91-101.

[83] J.-F. Wax, T. Bryk, J. Phys.: Condens. Matter, 25:32 (2013), 325104.

[84] Р. М. Хуснутдинов, А. В. Мокшин, Р. М. Юльметьев, ЖКЭТФ, 135:3 (2009), 477-489.

[85] Р. М. Хуснутдинов, А. В. Мокшин, Изв. РАН. Сер. физическал, 74 (2010), 677-680.

[86] R. M. Khusnutdinoff, A. V. Mokshin, I. I. Khadeev, J. Phys.: Conf. Ser., 394:1 (2012), 012012, 6 pp.

[87] Р. М. Хуснутдинов, А.В. Мокшин, И. И. Хадеев, Поверхностъ. Рентген., синхротр. и нейтрон. исслед., 2014, №1, 90-98.

[88] A. V. Mokshin, R. M. Yulmetyev, R. M. Khusnutdinoff, P. Hänggi, J. Phys.: Condens. Matter, 19:4 (2007), 046209, 16 pp.

[89] R. D. Mountain, Rev. Modern Phys., 38:1 (1966), 205-214.

[90] Л. Д. Ландау, Е. М. Лифшиц, Электродинамика сплошных сред, Наука, М., 1982.

[91] Г. С. Ландсберг, Оптика, Физматлит, М., 2003.

[92] И. Л. Фабелинский, УФН, 170:1 (2000), 93-108.

[93] W. Götze, "Aspects of structural glass transitions", Liquids, Freezing, and the Glass Transition, eds. J. P. Hansen, D. Levesque, J. Zinn-Justen, Elsevier, New York, 1991, 287-504.

[94] W. Götze, Complex Dynamics of Glass-Forming Liquids: A Mode-Coupling Theory, Oxford Univer. Press, Oxford, 2009.

Поступила в редакцию 13.08.2014 